\title{
SPATIALLY DEPENDENT HEATING AND IONIZATION IN AN ICME OBSERVED BY BOTH ACE AND ULYSSES
}

\author{
Susan T. LePri ${ }^{1}$, J. MARtin LAMing ${ }^{2}$, CARA E. RAKOWSKI $^{2}$, And Rudolf Von STEIGER ${ }^{3,4}$ \\ ${ }^{1}$ Department of Atmospheric, Oceanic and Space Sciences, University of Michigan, Ann Arbor, MI 48109-2143, USA \\ ${ }^{2}$ Space Science Division, Naval Research Laboratory, Code 7674L, Washington, DC 20375-5321, USA \\ ${ }^{3}$ International Space Science Institute, Bern CH-3012, Switzerland \\ ${ }^{4}$ Physikalisches Institut, University of Bern, Bern CH-3012, Switzerland \\ Received 2012 May 15; accepted 2012 September 26; published 2012 November 9
}

\begin{abstract}
The 2005 January 21 interplanetary coronal mass ejection (ICME) observed by multiple spacecraft at L1 was also observed from January 21-February 4 at Ulysses (5.3 AU). Previous studies of this ICME have found evidence suggesting that the flanks of a magnetic cloud like structure associated with this ICME were observed at L1 while a more central cut through the associated magnetic cloud was observed at Ulysses. This event allows us to study spatial variation across the ICME and relate it to the eruption at the Sun. In order to examine the spatial dependence of the heating in this ICME, we present an analysis and comparison of the heavy ion composition observed during the passage of the ICME at L1 and at Ulysses. Using SWICS, we compare the heavy ion composition across the two different observation cuts through the ICME and compare it with predictions for heating during the eruption based on models of the time-dependent ionization balance throughout the event.
\end{abstract}

Key words: solar wind - Sun: corona - Sun: coronal mass ejections (CMEs) - Sun: flares

Online-only material: color figures

\section{INTRODUCTION}

The solar wind and interplanetary coronal mass ejections (ICMEs, hereafter we will refer to the interplanetary manifestation of coronal mass ejections as ICMEs and refer to their solar counterpart as CMEs) escape a corona that is heated to temperatures typically $>1 \mathrm{MK}$. The physical reasons for this hot corona and the temperature profile in the accelerated heliospheric plasma are therefore at the center of interest to all solar and heliospheric physics. These elevated coronal temperatures are derived from electron observations in the corona and inferred from ionic charge states observed in the solar wind. There are important measurements of the electron temperature in the low corona up to $0.6 R_{\odot}$ from the solar surface (see, e.g., Ko et al. 2002; Wilhelm et al. 2002, and references therein). However, there is limited information about the coronal electron temperature beyond that point. The frozen-in ionic composition of solar wind heavy ions, which is determined by coronal conditions between $1.2 R_{\odot}$ and $4 R_{\odot}$ (Chen et al. 2003; Bame et al. 1974; Bürgi \& Geiss 1986), is therefore unique and a very important measure of the heating and expansion of the corona and the solar wind near the Sun. The composition data complement solar remote observations and link them to in situ plasma properties.

ICMEs are identified in situ by a number of plasma characteristics. Many signatures of ICMEs have been reported in previous statistical studies (e.g., Gosling 1990, and references therein; Neugebauer et al. 1997). Some of those signatures include the presence of counterstreaming suprathermal electrons indicating the presence of closed magnetic loops (Gosling 1990), specific velocity and density profiles (Gosling \& Riley 1996), certain magnetic field topologies (Burlaga 1991; Gosling 1990), and various compositional signatures such as He enhancements and anomalous charge states (Borrini et al. 1982, also see Zurbuchen \& Richardson 2006 for a detailed review). Several case studies and surveys have identified anomalously high ionization states in ICMEs (Fenimore 1980; Henke et al. 1998; Gloeckler et al. 1998, 1999; Zurbuchen \& Richardson 2006; Zurbuchen et al.
2004; Lepri \& Zurbuchen 2004a; Lepri et al. 2001; Henke et al. 2001; Galvin \& Gloeckler 1997; Reinard 2005). High Fe charge states have been found to be excellent sufficient signatures of ICMEs (Lepri et al. 2001). Once the ions freeze-in close to the Sun, their charge states remained fixed as they expand further into the heliosphere, making their properties independent of radial distance and plasma interactions (Hundhausen 1970). These characteristics make them ideally suited for the identification of ICMEs over a large range of heliospheric distances.

Significant variations in the observed ICME signatures have been seen in measurements from 1 AU (see Lepri \& Zurbuchen $2004 \mathrm{~b}$, and references therein). These variations may be due to spatial structures within the ICME plasma or due to the structure of the ICME source region (Antiochos 1998). ICMEs have been observed at $1 \mathrm{AU}$ that retain these spatially distinct particle populations (Haggerty et al. 2000; Skoug et al. 2000). As a result, the signatures observed may depend upon the observational cut through the ICME and its properties close to the Sun.

It is the purpose of this paper to examine a single ICME event which was observed by two separated spacecraft, $A C E$ and Ulysses, in order to characterize the spatial structure that exists within an individual event. In this unique study, we examine the ionization states observed in each observational cut through the ICME to study the heating conditions that were present during the eruption. Using an ionization model by Laming (2004) and Laming \& Lepri (2007), we will examine the partitioning of energy released during the eruption as well as the coronal temperatures in the source region and expansion characteristics of the different portions of the same ICME. This paper builds on the work of Rakowski et al. (2007) who examined ICMEs observed by $A C E$ and interpreted them in the context of the same ionization model, and Rakowski et al. (2011) who studied the 2007 May 19 CME observed by STEREO.

This paper is organized as follows. Section 2 describes the ICME, CME, and flare in question, through both in situ and imaging observations. Section 3 describes the element charge state observations in more detail, and Section 4 describes the 


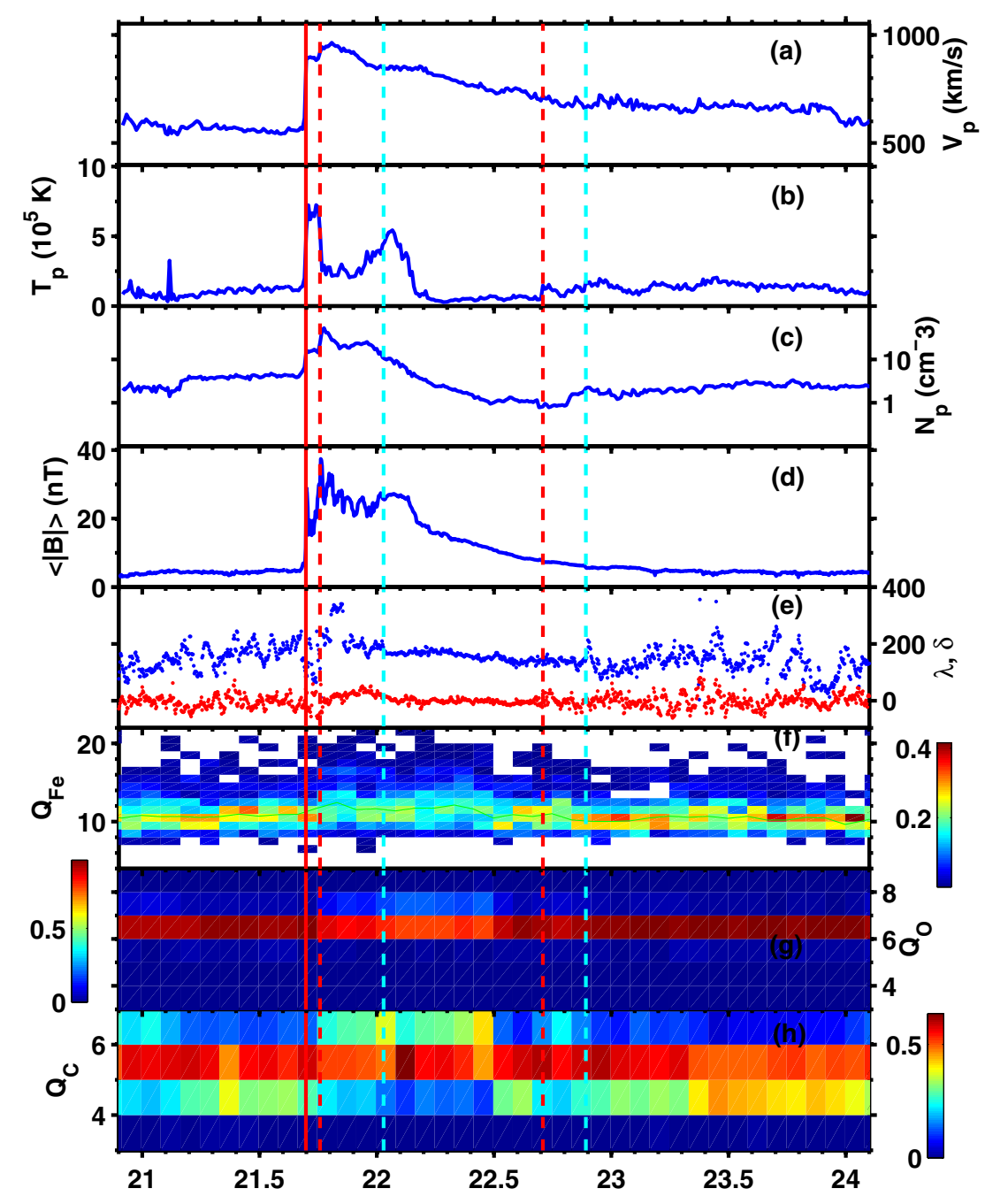

Figure 1. Solar wind conditions observed by $A C E$ during ICME passage. Panels are (a) proton velocity, (b) proton temperature, (c) proton density all derived from the merged ACE SWICS/SWEPAM level 3 data set, (d) magnetic field strength, (e) longitude and latitude angles of magnetic field direction, as measured by the $A C E$ MAG instrument, and (f-h) the charge state distributions for Fe, O, and C measured by the Solar Wind Ion Composition Spectrometer (SWICS). A shock preceding the ICME is show by the red solid line. The ejecta boundary is shown by the red dashed lines. The cyan dashed lines show the boundary of the magnetic cloud.

(A color version of this figure is available in the online journal.)

procedures for modeling these charge states. Section 5 discusses the results of such modeling, and Section 6 concludes.

\section{PROPERTIES OF THE JANUARY 21 ICME}

\subsection{The ICME}

The 2005 January 21 ICME event has been studied in detail using data from a large number of satellites. In particular, Foullon et al. (2007) conducted a multi-spacecraft study in an effort to understand the macroscopic structure, dynamics, and evolution of an ICME. This ICME event was associated with a well-studied large solar energetic particle event which occurred on 2005 January 20 (e.g., Mewaldt et al. 2005; Gopalswamy et al. 2005; Simnett 2006). They were able to establish that the same ICME was observed by ACE, Cluster, Geotail, WIND, and even Ulysses.

The ACE spacecraft, launched in 1997 , is currently in orbit about the L1 point, 1.5 million km sunward of Earth. ACE contains a suite of nine high-resolution sensors dedicated to measuring the heliospheric magnetic field, plasma properties, elemental, isotopic, and ionization properties (Stone et al. 1998). At 1 AU, according to Foullon et al. (2007), the ICME was observed by $A C E$ to be preceded by a shock and was determined to exhibit magnetic cloud like (MCL) features (e.g., possessing the same features of a magnetic cloud (MC) except not displaying the coherent magnetic field rotation). The ICME lasts from $\sim 1800$ UT on January 21 through $\sim 2100$ UT on January 22 as observed by $A C E$.

Figure 1 shows the solar wind conditions observed by $A C E$ during the passage of the ICME. Panel (a) shows the proton velocity, $V_{p}$, panel (b) the proton temperature, $T_{p}$, and panel (c) the proton density, $N_{p}$. These values are derived from the merged Solar Wind Ion Composition Spectrometer (SWICS)/ Solar Wind Electron, Proton, and Alpha Monitor (SWEPAM) data set available at the $A C E$ Science Center as an LV3 data product. Panel (d) shows the magnitude of the magnetic field and panel (e) shows the latitude and longitude angles of the field as measured by the MAG instrument. Panels $(\mathrm{f}-\mathrm{h})$ show the charge state distributions of $\mathrm{Fe}, \mathrm{O}, \mathrm{C}$, respectively, with $2 \mathrm{hr}$ cadence. Charge states are shown on the $y$ axis, and the color map shows the relative abundance of the individual charge states measured by SWICS. A shock that preceded the ICME is shown by the solid red line, while the ejecta boundary is shown by the dashed red lines. The MCL structure appears at the beginning 


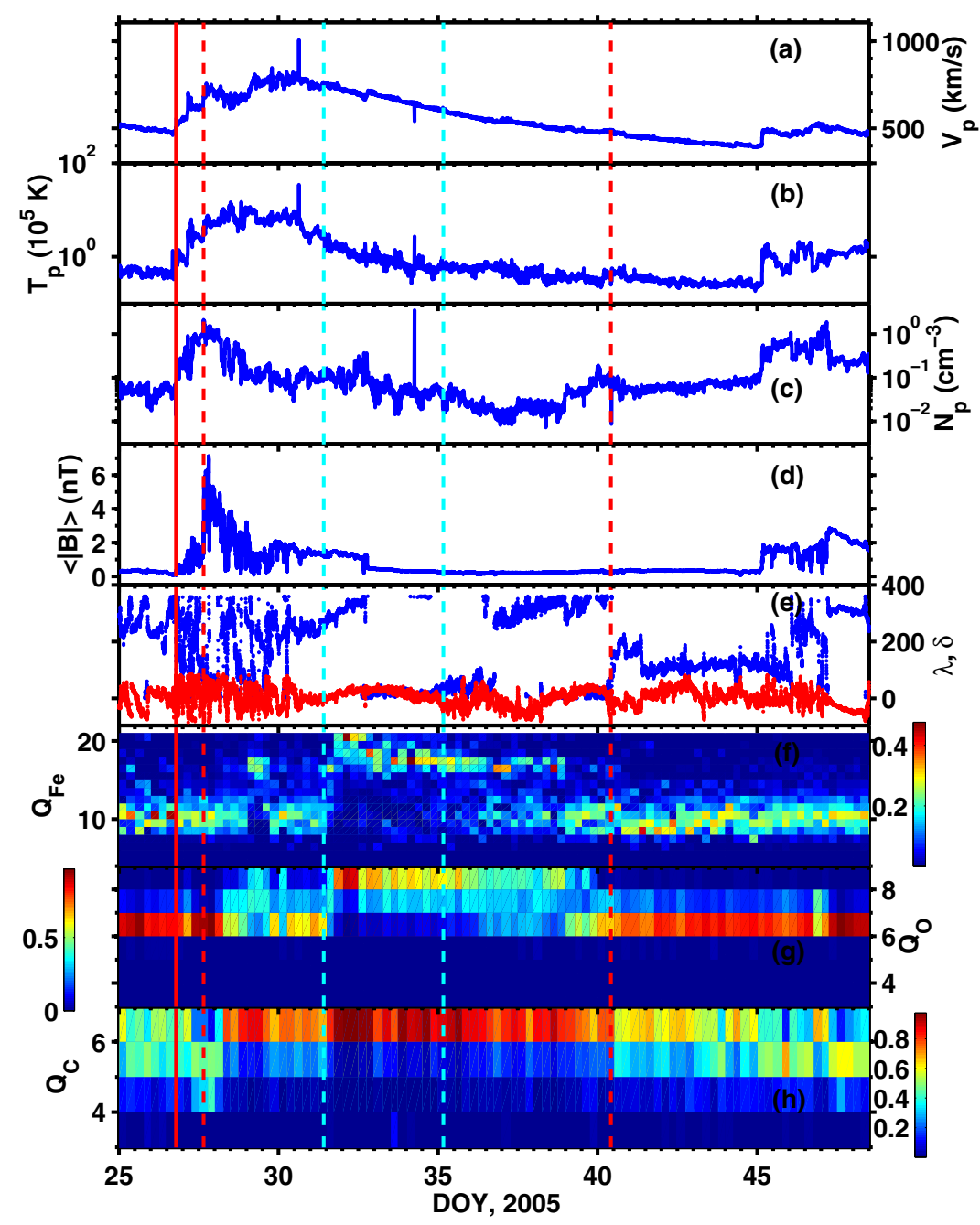

Figure 2. Solar wind conditions observed by Ulysses during ICME passage. Panels are (a) proton velocity, (b) proton temperature, (c) proton density, all determined by the Solar Wind Observations Over the Poles of the Sun (SWOOPS), (d) magnetic field strength, (e) longitude and latitude angles of magnetic field direction, as measured by the Vector Helium Magnetometer (VHM) instrument, and (f-h) charge state distributions for Fe, O, and C measured by the Solar Wind Ion Composition Spectrometer (SWICS). A shock preceding the ICME is show by the red solid line. The ejecta boundary is shown by the red dashed lines. The cyan dashed lines show the boundary of the magnetic cloud. Other features are as in Figure 1. The smooth rotation of the magnetic field in the magnetic cloud is absent in Figure 1.

(A color version of this figure is available in the online journal.)

of DOY 22 and persists through most of the day and is bounded by the dashed cyan lines. The ICME is characterized by the monotonically decreasing velocity profile, a proton temperature depression during DOY 22, enhanced density and magnetic fields and steady, smooth magnetic field angles. The charge state signatures are not dramatically shifted toward higher charge states at $A C E$. A tail that extends toward higher charge states becomes more evident during the ICME, but is somewhat subtle. A green line tracks the average charge state of $\mathrm{Fe}$ in panel (f); it shifts up slightly during the ICME. Although not shown, counterstreaming suprathermal electrons $(272 \mathrm{eV})$ were observed during the first half of the MCL. For a more detailed discussion of the ejecta properties, the reader is referred to the Foullon et al. (2007) paper. The subtle composition signatures and the MCL structure support the idea that $A C E$ passed through the flanks of the ICME.

Ulysses is a joint mission between the NASA and the European Space Agency (ESA). It was launched in 1990 and was injected into an elliptical orbit about the Sun's poles. Ulysses orbits the Sun at a distance that ranges from 1.4 to $5.4 \mathrm{AU}$ and reaches latitudes up to $\pm 80^{\circ}$ during its polar passes. The

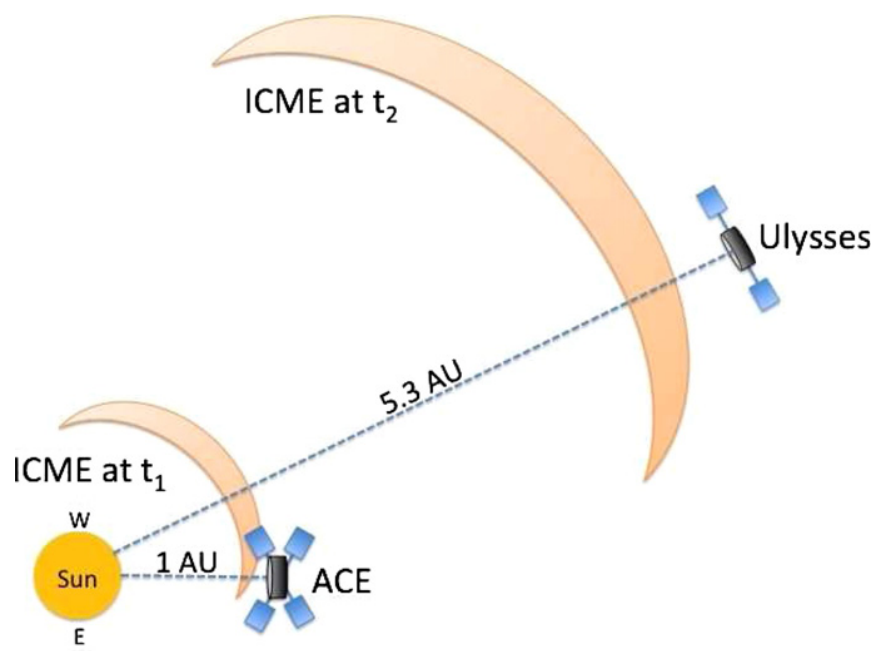

Figure 3. Schematic diagram of ICME evolution and interactions with the $A C E$ and Ulysses spacecraft. ACE intercepts the flank of the ICME at $1 \mathrm{AU}$, while Ulysses penetrates a more central portion later on at $5.3 \mathrm{AU}, 17^{\circ}$ south of the ecliptic, and $29^{\circ}$ west of the Earth in longitude.

(A color version of this figure is available in the online journal.) 


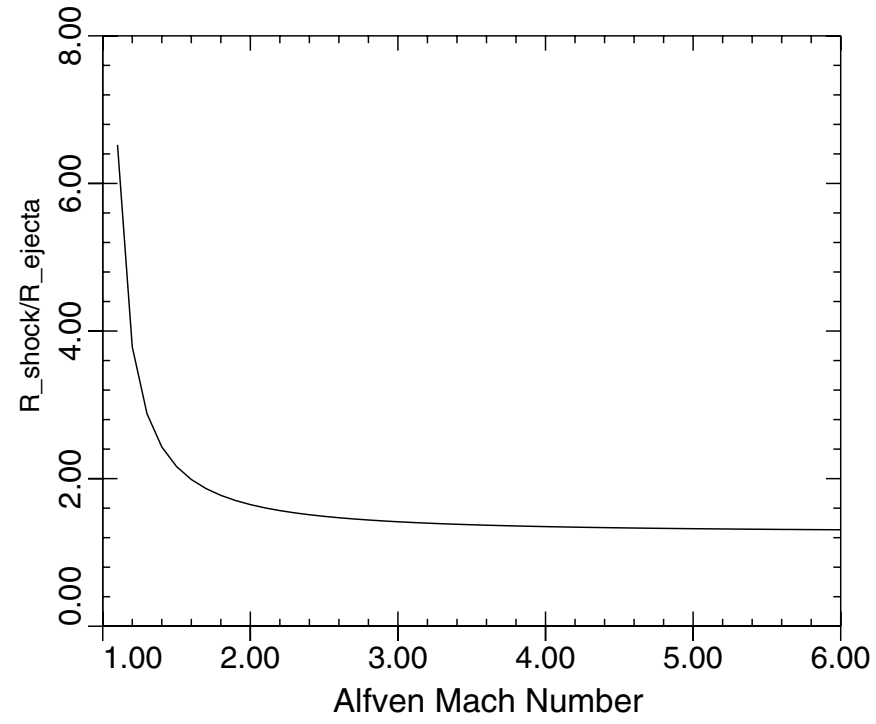

Figure 4. Ratio of shock radius to magnetic cloud radius as a function of shock Alfvén Mach number, calculated from Equation (1), with Equations (4) and (5).

Ulysses spacecraft contains a variety of sensors including those that study the interplanetary magnetic field, energetic particles, the solar wind plasma, and ionic composition (see Wenzel et al.
1992). An MC ICME was observed at Ulysses at 5.3 AU at $27^{\circ}$ westward from the Sun-Earth line (Foullon et al. 2007). This MC was determined to be part of the same ICME observed at 1AU. The ICME began on 2005 January 26 and lasted through February 9. Figure 2 shows the solar wind conditions observed by Ulysses during the passage of this ICME. The panels are the same as described in Figure 1. Proton density, temperature, and velocity were obtained from the Solar Wind Observations Over the Poles of the Sun (SWOOPS) sensor, magnetic field parameters from the Vector Helium Magnetometer (VHM) and the charge state distributions were obtained from SWICS on Ulysses. Here the time cadence of the charge state observations is $6 \mathrm{hr}$ to ensure sufficient statistical accuracy at the larger heliocentric distance of Ulysses. The preceding shock is shown by the solid red line, the ejecta boundaries are shown by the dashed red lines, and the MC boundaries are denoted by the dashed cyan lines. The ICME at Ulysses shows a similar monotonically decreasing velocity profile and exhibits enhanced densities and magnetic fields in the pile-up region near the front of the ICME. The magnetic field vectors exhibit the smooth rotations that are characteristic of a clear MC. Analysis by Foullon et al. (2007) and Rodriguez et al. (2008) of magnetic data, fitting flux rope models to the components of the magnetic field, reveals that $A C E$ observed the flank of the ICME whereas Ulysses observed a more central cut of the ICME. The scenario

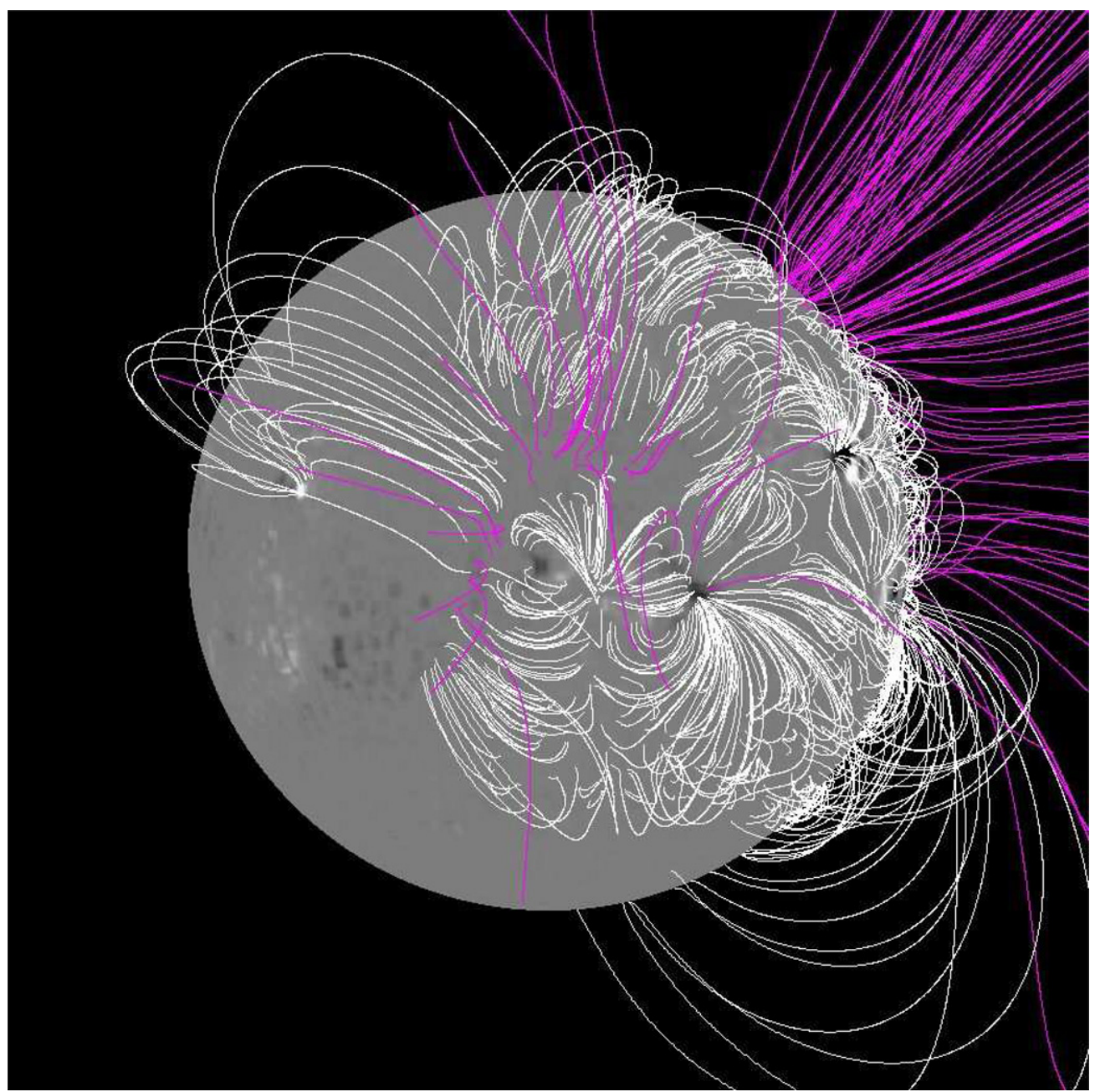

Figure 5. Potential field source surface (PFSS) extrapolation of photospheric magnetic field from 2005 January 20, 06:04:00. (A color version of this figure is available in the online journal.) 


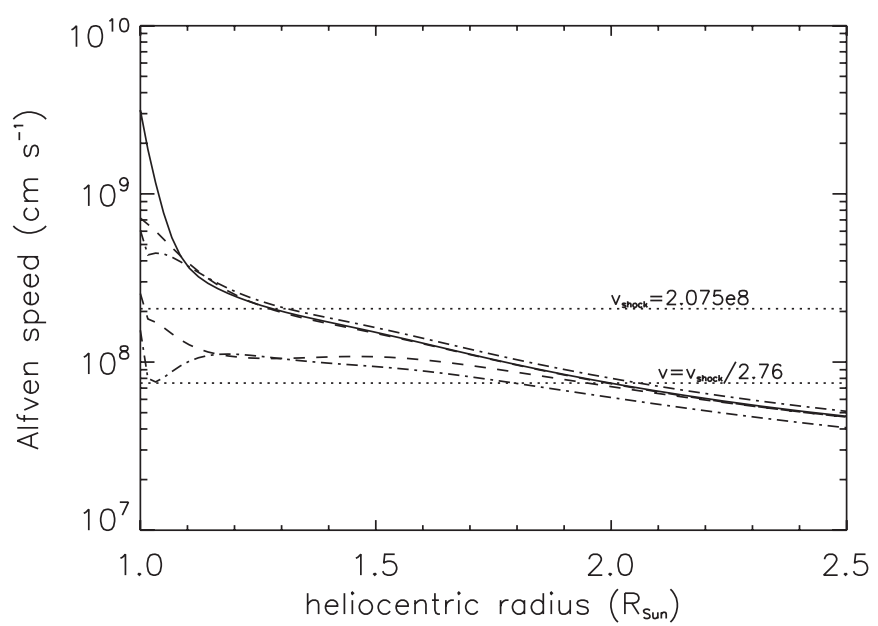

Figure 6. Plots of variation of Alfvén speed with heliocentric radius. The solid curve is at longitude $\mathrm{W} 61^{\circ}$, latitude $\mathrm{N} 14^{\circ}$ (Cane et al. 2006), on the active region at the center of the extrapolation. Dashed curves show the variation of $\pm 10^{\circ}$ in longitude $\left(+10^{\circ}\right.$ upper, $-10^{\circ}$ lower $)$, and the dash-dotted curves show the variation of \pm 9.35 in latitude ( +9.35 upper, -9.35 lower). The horizontal dotted lines show the shock velocity, $2075 \mathrm{~km} \mathrm{~s}^{-1}$ from Grechnev et al. (2008), and the shock velocity divided by 2.76 . The intersection of this last line with the Alfvén speed curves shows the location where the shock becomes supercritical and begins to accelerate particles.

is illustrated schematically in Figure 3. ACE intercepted the edge of the ejected plasma at $1 \mathrm{AU}$, while further out at 5.3 $\mathrm{AU}$, and $29^{\circ}$ to the west, Ulysses sees a more central portion. The ICME itself was associated with a flare even further to the west, at $\mathrm{W} 61^{\circ}$ (Cane et al. 2006; Rodriguez et al. 2008). As we shall see, the composition measurements also support this conclusion. Ulysses detected significantly higher element charge states, which are unmistakably related to material heated to very high temperatures in the ejection from the Sun, and which are more plausibly located in central regions of the ICME.

\subsection{The Flare and $C M E$}

The associated flare and coronal mass ejection (CME) are studied by Grechnev et al. (2008). They present images in different wavebands from RHESSI and TRACE of the flare evolution, and track the resulting wave that eventually develops into a shock in EIT $195 \AA$ and LASCO C2 and C3. For the 2005 January 20 event, the shock velocity reaches $2000 \mathrm{~km} \mathrm{~s}^{-1}$ by a radius of $5 R_{\odot}$ at a position angle of $290^{\circ}$ (in the NW). For our modeling, we need some inference on the motion not of the shock itself, but of the CME ejecta following it. It is crucial to the conclusions of this paper that the evolution of the ejecta density, through their expansion velocity and radius, be as accurate as possible. This determines where ion charge states freeze in, and consequently the charge states ultimately detected by $A C E$ and Ulysses.

Gopalswamy \& Yashiro (2011) have used the observed stand-off distance between a CME shock and flux rope to estimate the magnetic field in the outer corona. We adopt a variation on this approach, using a potential field source surface (PFSS) extrapolation to estimate the magnetic field, and hence with a model for the coronal density, the Alfvén Mach number of the CME. This Mach number can then be related to the stand-off distance, and hence the difference in shock and flux rope expansion velocities, in the following manner. Russell \& Mulligan (2002), following Farris \& Russell (1994), conjecture that the ratio of shock to flux rope heliocentric radii
$\left(R_{\text {shock }} / R_{\text {ejecta }}\right)$ can be written

$$
\frac{R_{\text {shock }}}{R_{\text {ejecta }}}=1+1.1 \frac{M_{2}^{2}}{1-M_{2}^{2}},
$$

where $M_{2}$ is the post-shock Mach number. The above authors all take $M_{2}$ to be the sonic Mach number. We argue that it should more properly be a magnetosonic post-shock Mach number, especially for CME evolution close to the Sun where the shock is most likely a quasi-perpendicular magnetohydrodynamic (MHD) shock (e.g., Rouillard et al. 2011). Further out the shock is believed to evolve toward a quasi-parallel geometry, where $M_{2}$ may more appropriately be taken as a sonic Mach number. Savani et al. (2012) investigate the variation of the constant (1.1 in Equation (1)) with differing geometry and heliocentric distance.

We will approximate the CME shock as a perpendicular shock. The MHD energy equation then yields

$$
\frac{3}{M_{s 2}^{2} r^{2}}=1+\frac{2}{M_{A 1}^{2}}-\frac{2}{M_{A 2}^{2} r^{2}}+\frac{3}{M_{s 1}^{2}}-\frac{1}{r^{2}},
$$

where $M_{s, A}$ denote sonic and Alfvén Mach numbers, subscripts 1 and 2 denote pre- and post-shock quantities, and $r$ is the shock compression. In the limit $M_{s 1} \rightarrow \infty$,

$$
M_{s 2}^{2}=\frac{3 / r^{2}}{1+2(1-r) / M_{A 1}^{2}-1 / r^{2}}
$$

and

$$
r=\sqrt{\left(M_{A 1}^{2}+5 / 2\right)^{2}+8 M_{A 1}^{2}}-\left(M_{A 1}^{2}+5 / 2\right),
$$

which give $M_{s 2}>1$ for $M_{A 1}<2.76$ (Edmiston \& Kennel 1984), which would clearly be incompatible with Equation (1) if $M_{2}=M_{s 2}$. The post-shock magnetosonic Mach number $M_{M S 2}$ is given by

$$
M_{M S 2}^{2}=\frac{1}{M_{A 2}^{-2}+M_{s 2}^{-2}}=\frac{3 M_{A 1}^{2}}{M_{A 1}^{2}\left(r^{2}-1\right)+2 r^{2}+r^{3}} .
$$

In Figure 4 we plot the relationship given by Equation (1) against $M_{A 1}$, with $M_{2}=M_{M S 2}$ as given by Equation (5). Figure 5 shows a PFSS magnetic field extrapolation, performed using the PFSS package in SolarSoft, which follows Schrijver (2001) and Schrijver \& DeRosa (2003). It is centered on the active region from where the 2005 January $20 \mathrm{CME}$ erupted, and based on a magnetogram taken at 06:04:00 on that date. Closed field lines are shown in white, and open field lines in purple. This extrapolation is used to calculate the radial profile of the Alfvén speed, $V_{A}$, at various locations around the active region, which are shown in Figure 6. The solid line shows the radial profile of $V_{A}$ at latitude $\mathrm{N} 14^{\circ}$ and longitude $\mathrm{W} 61^{\circ}$, the location of the associated flare (Cane et al. 2006; Rodriguez et al. 2008). The dashed curves show the variation with $\pm 10^{\circ}$ in longitude, and the dash-dotted curves the variation with \pm 9.35 in latitude. The horizontal dotted lines show the shock speed of $2075 \mathrm{~km} \mathrm{~s}^{-1}$ from Grechnev et al. (2008), and this speed divided by the first critical Alfvén Mach number (with value 2.76 for a perpendicular shock in low $\beta$ plasma; Edmiston \& Kennel 1984). The intersection of the lower line with the curve for Alfvén speed shows the heliocentric radius at which the CME shock should become supercritical and begin 

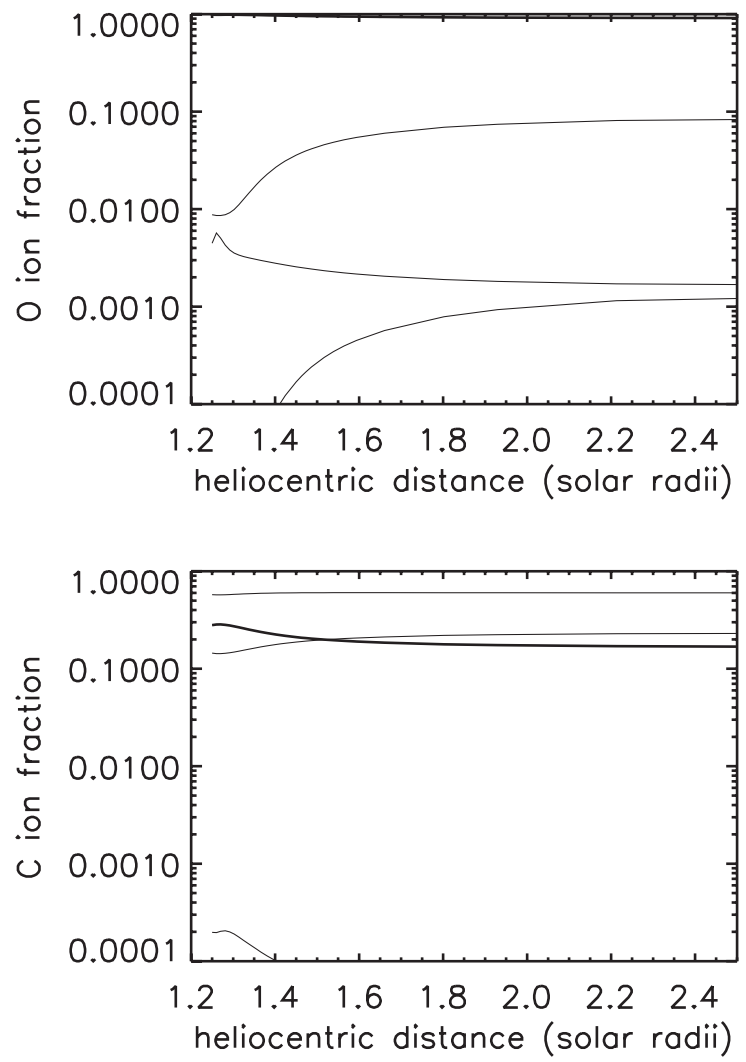
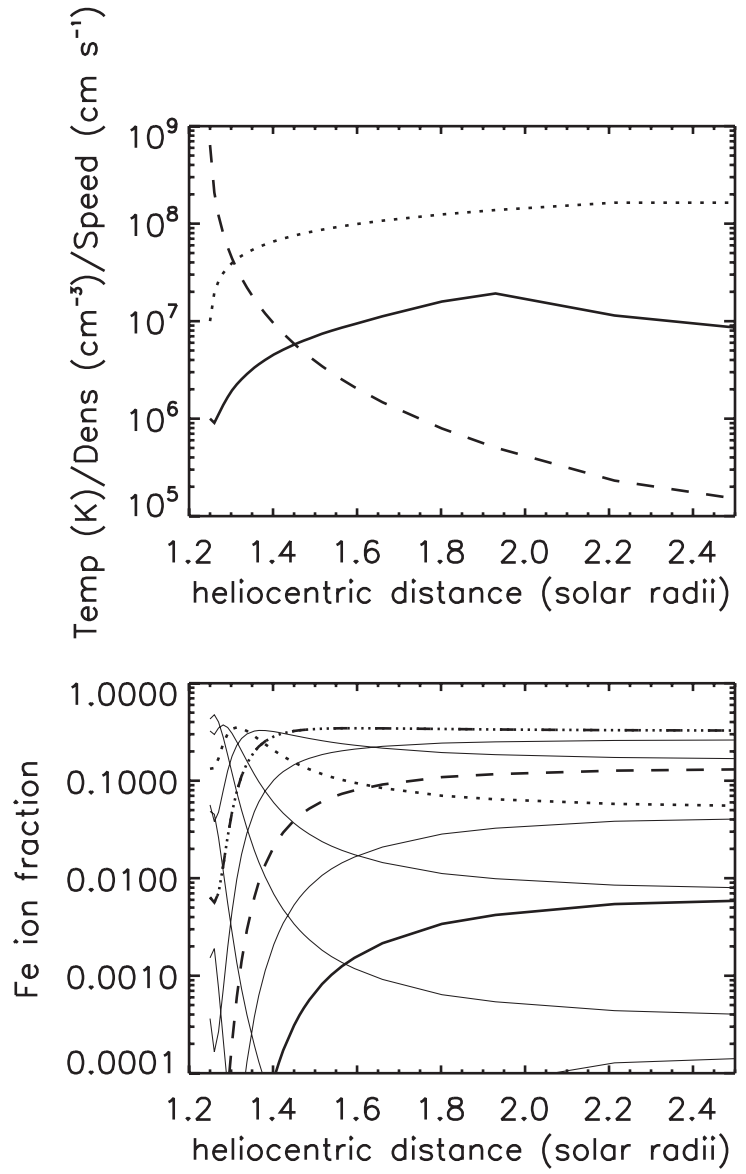

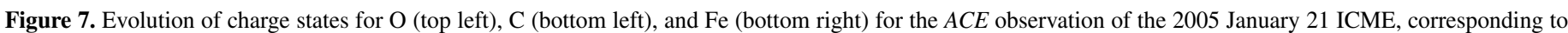

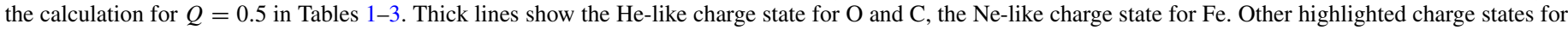

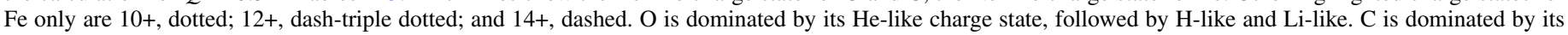
$\mathrm{H}$-like ion. Top right shows the plasma temperature (solid line), density (dashed line), and expansion velocity (dotted line).

to accelerate particles. The value we infer, about $2 R_{\odot}$, agrees well with the onset of $\pi^{0}$ decay emission and the ground level event (Figure 14, Grechnev et al. 2008) and the extrapolated shock radius at this time (Figure 13, Grechnev et al. 2008). Interestingly, Grechnev et al. (2008) argue that these and the other electromagnetic emissions they discuss come from the flare itself and not the shock, though this point makes little difference to the arguments in this paper.

With this estimate of the Alfvén Mach number of the forward shock, we can infer the heliocentric radius of the flux rope from the radius of the forward shock using the relation plotted in Figure 4, and estimate the expansion speed of the flux rope from this ratio also.

\section{ELEMENT CHARGE STATE OBSERVATIONS}

The data that $A C E$ provides have been essential in enabling the identification and study of ICMEs (e.g., Zurbuchen \& Richardson 2006; Zurbuchen et al. 2003, 2004; Burlaga et al. 2001; Lepri et al. 2001; Reinard et al. 2001; Cane \& Richardson 2003; Gloeckler et al. 1999; Farrugia et al. 2002; Lynch et al. 2003).

Of particular importance to this study is the SWICS which comprehensively measures the ionic composition of the solar wind (Gloeckler et al. 1998). SWICS is a time-of-flight mass spectrometer paired with energy-resolving solid-state detectors (SSDs) and an electrostatic analyzer (ESA). Ions are mass and speed (or energy and charge) selected in the ESA and the residual energy measured by the SSDs enables particle identification. A triple coincidence technique is employed which allows the determination of mass $M$, charge $Q$, and energy $E$ to all be virtually free of background contamination. Data are corrected for event priorities, instrument efficiency, and duty cycle. Fe charge states between 6 and 24 are identified in the energy per charge range of $0.49-60 \mathrm{keV} e^{-1}$, which is in the thermal energy range of the solar wind. Ulysses contains a SWICS instrument virtually identical in design and operation to the SWICS instrument on $A C E$, however its energy per charge range extends up to $100 \mathrm{keV} e^{-1}$ (Gloeckler et al. 1992). The presence of virtually the same instrument on both spacecraft allows for reliable two-point observations of the ion composition from multiple heliospheric latitudes and longitudes.

In Figures 1 and 2 we can see that the ejecta do contain hot ICME material, which is not exclusively confined to the MCL or MC period. Ulysses observed the ICME at a later date than $A C E$ when the ejecta had expanded further into the heliosphere. The charge states of Fe in panel (f) in Figure 2 show evidence for more highly ionized material within the $\mathrm{MC}$ cross-section of the ejecta than is the case with $A C E$ (panel (f) in Figure 1). The charge states remain elevated for nearly 10 days and reach a maximum of around $\left\langle Q_{\mathrm{Fe}}\right\rangle \geqslant 16$. Minimum variance analysis of the MC and ejecta by Foullon et al. (2007) and by Rodriguez et al. (2008), discussed above, supports the idea that this cut through the ICME was much closer to the center. Reinard (2008) also sees higher charge states in the more central portions of a 

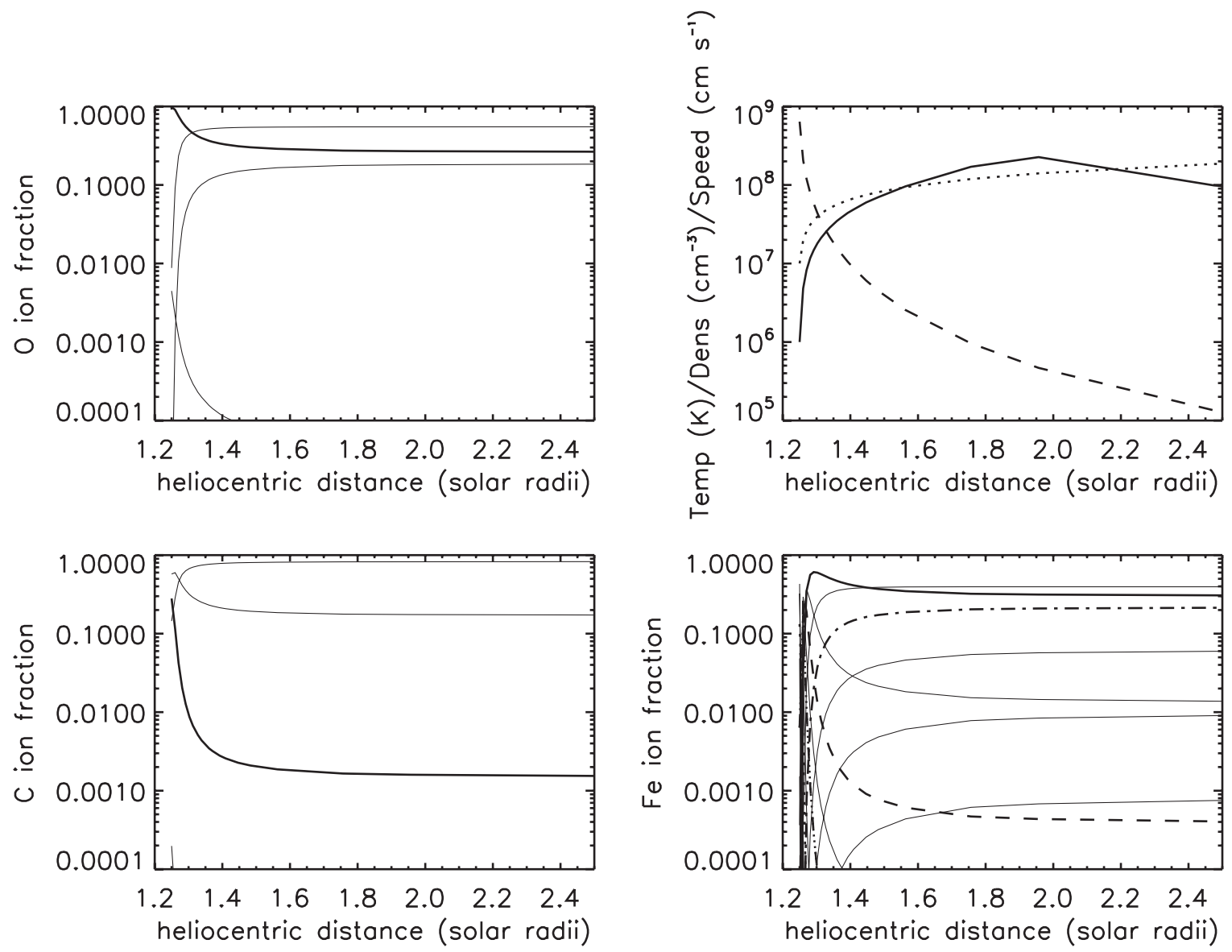

Figure 8. Same as Figure 6, but for the Ulysses ICME observation. The charge states correspond to the $Q=5.0$ entries in Tables 1-3. An extra charge state of Fe is highlighted; $18+$ is shown by the dash-dotted line. $\mathrm{Fe}^{17+}$ is the most abundant ion. $\mathrm{O}$ is dominated by its $\mathrm{H}$-like charge state, followed by the He-like, and $\mathrm{C}$ is almost completely ionized.

sample of CMEs observed by $A C E$, as do Lynch et al. (2011) in their flux-cancellation CME simulation. Interestingly, Lynch et al. (2011) do not see such elevated Fe charge states in their faster break-out CME simulation. Reinard (2008) also sees Fe charge states relatively insensitive to the associated flare size, though the ratio $\mathrm{O}^{7+} / \mathrm{O}^{6+}$ clearly correlates. Berkebile-Stoiser et al. (2012) also see a strong correlation between CME peak velocity and the total energy in accelerated electrons.

\section{IONIZATION AND FREEZE-IN MODEL}

Charge states of solar wind ions are determined close to the Sun, freezing in between 1.2 and 4 solar radii. The freeze-in concept of heavy ions during their transit out of the corona is straightforward (Hundhausen 1968; Bame et al. 1974; Owocki et al. 1983). In the near corona, where ions and electrons are in near-collisional equilibrium, the charge distribution of ions reflects the local electron temperature. At increasing solar distances, the timescale for equilibration increases and, for any given ionization state of any element, freezes in where the expansion timescale is approximately equal to its recombination timescale. Hence, each ionization state has a freeze-in location that depends on its recombination rates. The location strongly depends on the specific electronic combinations (e.g., Bryans et al. 2006). For example, the Ne-like $\mathrm{Fe}^{16+}$ freezes in very late and therefore measures the temperature in a later stage of the expansion, or at a larger heliocentric radius, than $\mathrm{C}^{5+}$, which freezes in very close to the Sun (Geiss et al. 1995).
A large combination of freeze-in states therefore provides important constraints on the heating and expansion of solar wind heavy ions and hence the solar wind (Bürgi \& Geiss 1986; Esser \& Edgar 2001, 2002). Recent advances in the analysis techniques for the solar wind composition measured on $A C E$ and Ulysses have provided the most comprehensive data set on ionic composition to date, giving us the unique ability to determine very solid constraints on the heating and acceleration of ICMEs.

The ionization model tracks a Lagrangian plasma parcel along its MHD evolution as long as the density and temperature history are known. As in Rakowski et al. (2007), an adaptation of the BLASPHEMER (Blast Propagation in Highly Emitting Environment) code (Laming \& Grun 2002, 2003; Laming \& Hwang 2003; Laming 2004) is used to perform time-dependent ionization and track temperatures as the ICME expands into the heliosphere.

The creation and destruction of an ion species $X^{i}$ is governed by the following rate equation:

$$
\begin{aligned}
\frac{d n_{i}}{d t}= & -C_{i} n_{i} n_{e}-\left(R_{\mathrm{dr}, i}+R_{\mathrm{rr}, i}\right) n_{i} n_{e}+C_{i-1} n_{i-1} n_{e} \\
& +\left(R_{\mathrm{dr}, i+1}+R_{\mathrm{rr}, i+1}\right) n_{i+1} n_{e},
\end{aligned}
$$

where $n_{i}$ is the ion density, $n_{e}$ is the electron density, $C_{i}$ is the collisional ionization rate, $R_{\mathrm{dr}, i}$ is the dielectric recombination rate, and $R_{\mathrm{rr}, i}$ is the radiative recombination rate. These rates depend on the local electron temperature and the speed of the ion and are based on Bryans et al. (2006) with updates for 

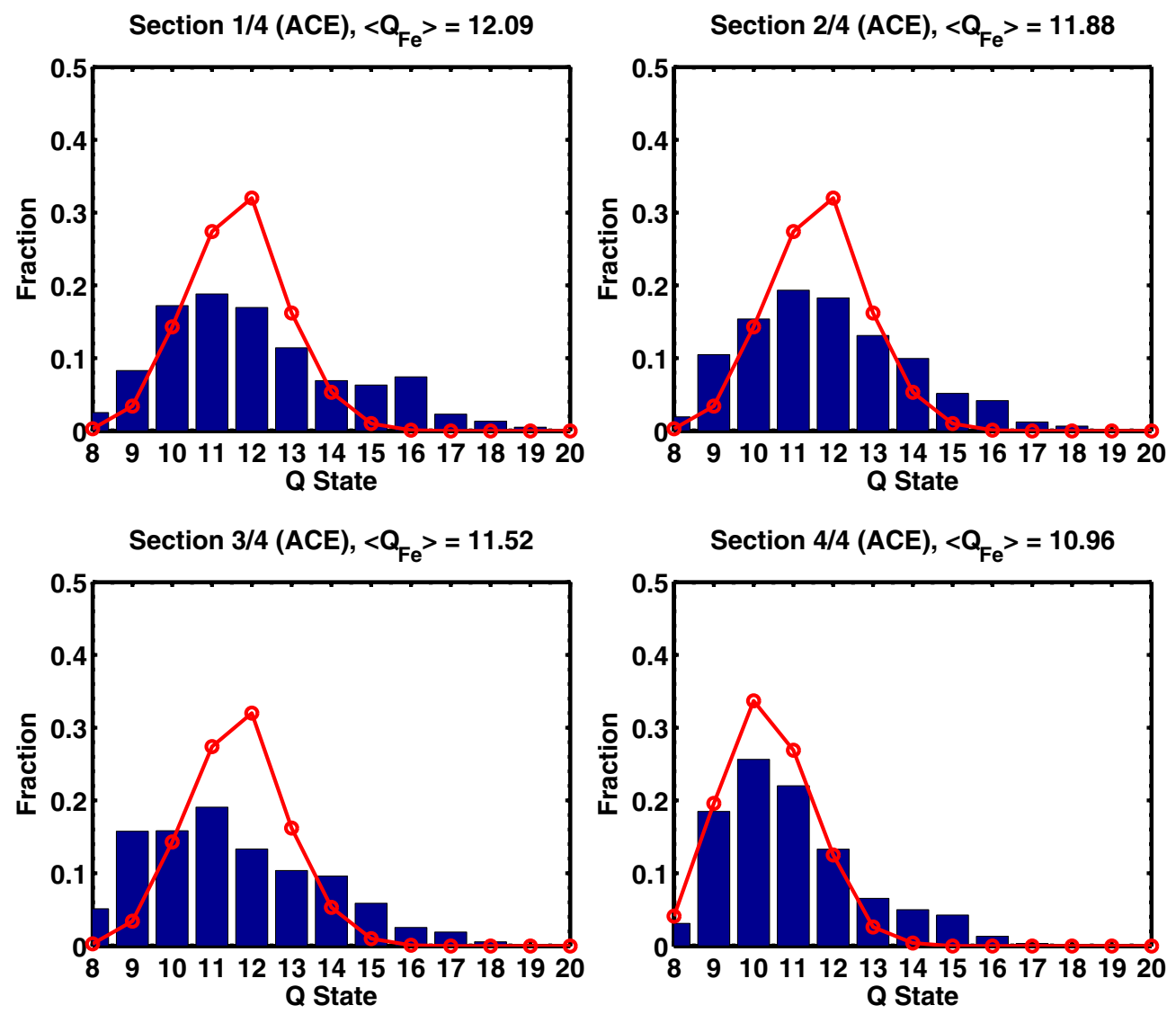

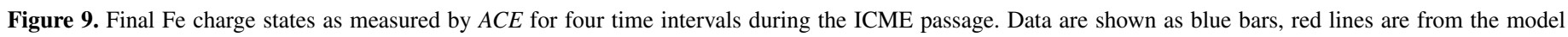
charge states.

(A color version of this figure is available in the online journal.)

various dielectric recombination rates as detailed in Rakowski et al. (2007).

Initially, we assume ionization equilibrium with temperatures ranging from $1 \times 10^{6} \mathrm{~K}$ representing typical coronal conditions to $5 \times 10^{6} \mathrm{~K}$ representing an active region environment. We begin with an initial electron density of $7 \times 10^{8} \mathrm{~cm}^{-3}$ at an initial flux rope heliocentric radius of $1.25 R_{\odot}$. This then gives the observed densities at $A C E$ and Ulysses after propagation and expansion to 1 and 5.3 AU, respectively. The initial speed of the ICME is assumed to be $100 \mathrm{~km} \mathrm{~s}^{-1}$ at $1.25 R_{\odot}$ and it reaches a peak speed of $1500 \mathrm{~km} \mathrm{~s}^{-1}$ at $2.5 R_{\odot}$, which is close to the observed speeds. Acceleration is assumed to be $2 \mathrm{~km} \mathrm{~s}^{-2}$ and is such that the CME reaches its final speed before $10 R_{\odot}$. Note that here we are interested in the expansion speed of the CME ejecta, which is inferred from the measured speed of the CME shock front and Equation (1), assuming $M_{2}=M_{M S 2}$ from Equation (5). The initial Alfvén speed inside the flux rope is assumed to be $1500 \mathrm{~km} \mathrm{~s}^{-1}$, evolving as $\rho^{1 / 6}$ as in Rakowski et al. (2007). Its magnitude is similar to the preshock Alfvén speed shown in the plots in Figure 5 coming from the magnetic field extrapolations. Additional heating during acceleration is modeled by a heating rate which is proportional to the increase in kinetic energy, with constant of proportionality $Q$, occurring during the acceleration phase. Berkebile-Stoiser et al. (2012) also observe strong correlation between energy in accelerated electrons and CME acceleration, supporting our approach.

The simplest model for ion charge states starts with plasma at a temperature of $1 \times 10^{6} \mathrm{~K}$, followed by heating as prescribed above during the eruption of the CME. Table 1 gives results for Fe charge states for different amounts of heating expressed as a fraction or multiple of the kinetic energy gain of the CME flux rope for two different initial temperatures. Charge state distributions peaking around $\mathrm{Fe}^{10+}$ or $\mathrm{Fe}^{12+}$ are found for thermal energy inputs of $0.25-0.5$ times the kinetic energy input starting at $1 \mathrm{MK}$. Higher charge states, $\mathrm{Fe}^{15+}$ and $\mathrm{Fe}^{16+}$, dominate when heating is about 1.0-2.0 times kinetic energy gain, and $\mathrm{Fe}^{18+}$ at 7.0-10.0 times higher. Also shown are charge states for selected heating rates for an initial temperature of $3 \mathrm{MK}$.

Tables 2 and 3 show the oxygen and carbon charge states from the same models at the same two initial temperatures. These are less variable than the Fe distributions. The dominant oxygen charge state is $\mathrm{O}^{6+}$ until the heating is at least five times the kinetic energy input for $1 \mathrm{MK}$, and $\mathrm{O}^{8+}$ dominates for the higher initial temperature at $3+$ times the kinetic energy input. Similarly, $\mathrm{C}^{5+}$ dominates for heating less than twice the kinetic energy, with $\mathrm{C}^{6+}$ predominant above that. $\mathrm{C}^{6+}$ dominates the higher temperature plasma.

Figures 7 and 8 show sample charge state evolutions for the portions of the CME observed by ACE and Ulysses, respectively. The calculations illustrated come from Tables 1-3, being the $Q=0.5$ and $Q=5$ cases, respectively, assuming an electron temperature of $1 \mathrm{MK}$. The top right panel in each case shows the radial evolution of the plasma temperature (solid line), the density (dashed line), and the CME expansion speed (dotted line). The remaining panels show the evolution of the charge state distributions of $\mathrm{O}, \mathrm{C}$, and $\mathrm{Fe}$ with heliocentric distance. In the cases of $\mathrm{O}$ and $\mathrm{C}$, the He-like charge state is highlighted in 

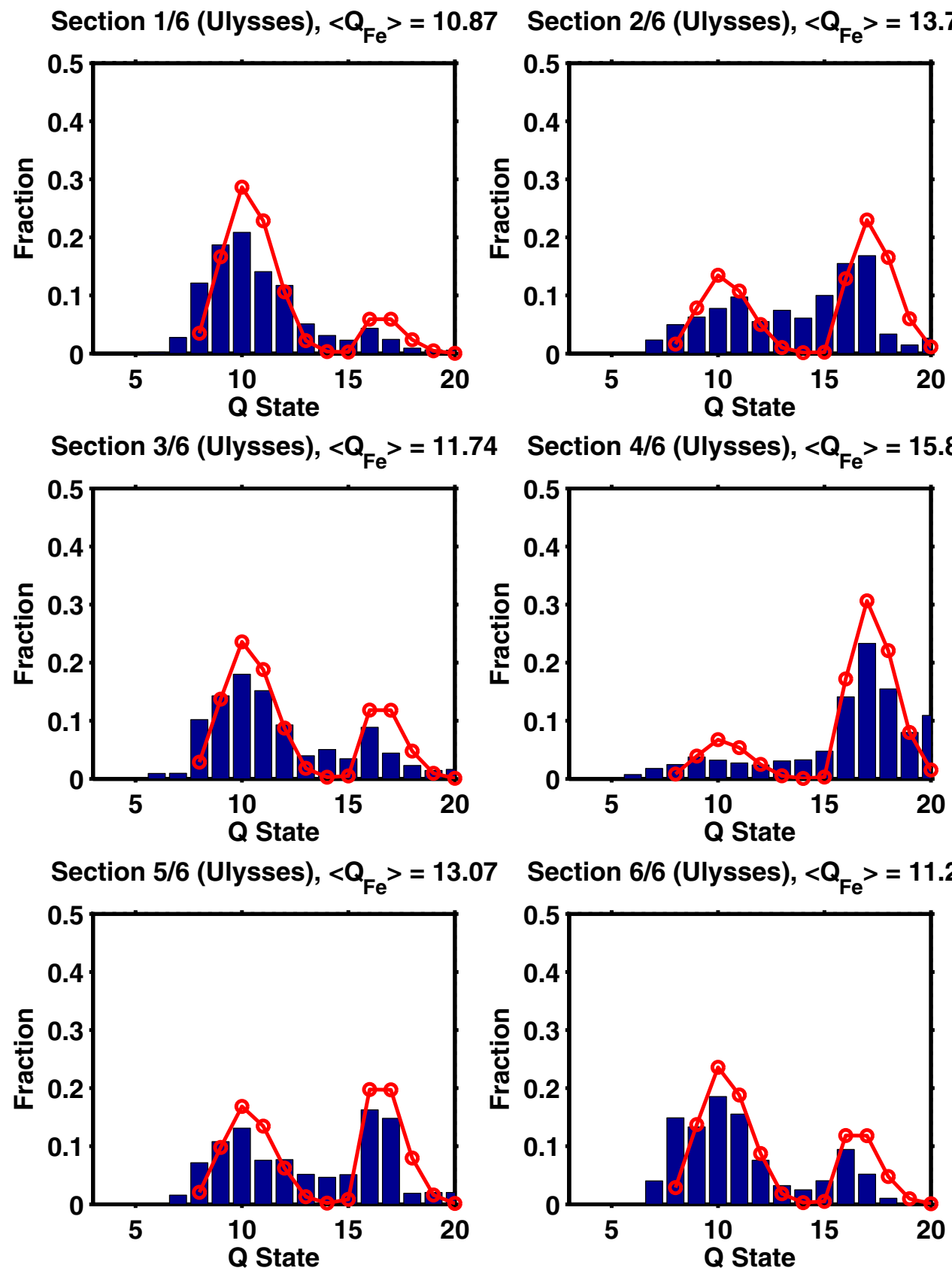

Figure 10. Same as Figure 8, but for six intervals during the Ulysses ICME observation. (A color version of this figure is available in the online journal.)

bold, for Fe it is the Ne-like charge state that is highlighted in this way. Other Fe charge states highlighted are $\mathrm{Fe}^{18+}$, dash-dotted; $\mathrm{Fe}^{14+}$, dashed; $\mathrm{Fe}^{12+}$, dash-triple dotted; and $\mathrm{Fe}^{10+}$, dotted. In Figure 7, $\mathrm{O}$ is dominated by the He-like charge state, $\mathrm{C}$ by the $\mathrm{H}$-like, and $\mathrm{Fe}$ is mainly $\mathrm{Fe}^{12+}$. In Figure $8 \mathrm{O}$ and $\mathrm{C}$ are mainly completely ionized (in the case of $\mathrm{C}$, this line is indistinguishable from the upper axis), and Fe is mainly Ne-like.

\section{MODEL RESULTS}

In order to interpret the ICME charge states, we divided the ICME observations into four equal parts in time at $A C E$. This was done so that some temporal variation would be preserved throughout the event, yet we had a manageable number of sections to model. Since the ICME at $A C E$ lasts only $20.25 \mathrm{hr}$, we divided the observations up into $6.75 \mathrm{hr}$ intervals. The ICME observed at Ulysses lasts longer and has more varied charge states. For this reason, we divide the ICME instead into six sections (as shown in Table 4) according to regions with similar charge state distributions.

Figure 9 shows the Fe charge state distributions (blue bars) in the four sections of the ICME observed by ACE SWICS. All sections show a broad distribution of charge states peaking at $\mathrm{Fe}^{10+}$ or $\mathrm{Fe}^{11+}$ and extending as high as $\mathrm{Fe}^{17+}$, but with small contributions of the higher charge states. Section 1 shows a mild bimodality with a second peak at $\mathrm{Fe}^{16+}$. Note the detail that can be seen in the charge state distributions compared to that seen in the average charge state (which shows only mild enhancements in the average value). The ionization model that 

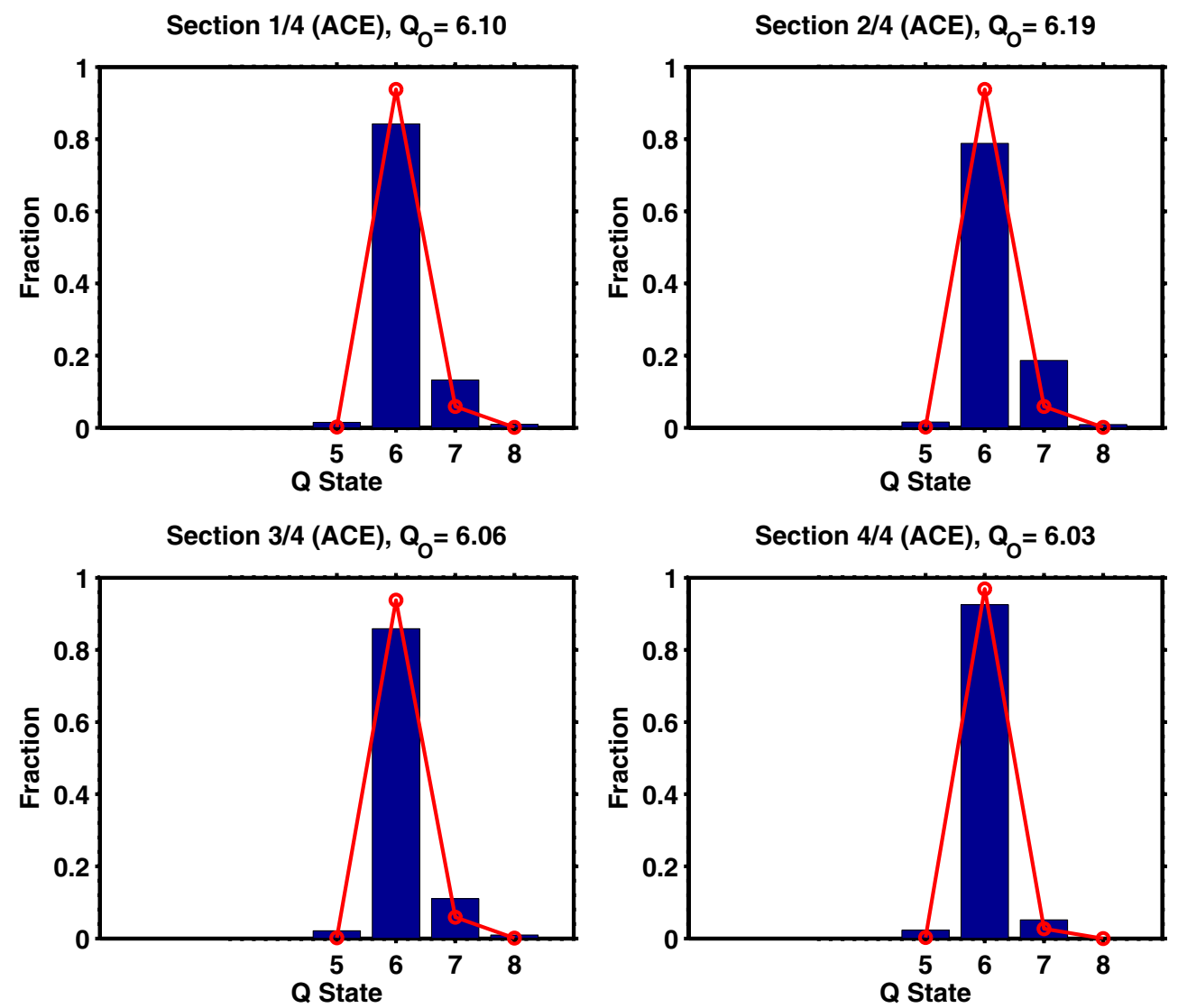

Figure 11. Final $\mathrm{O}$ charge states as measured by $A C E$ for four time intervals during the ICME passage.

(A color version of this figure is available in the online journal.)

Table 1

Iron Charge States with Heating During the Eruption

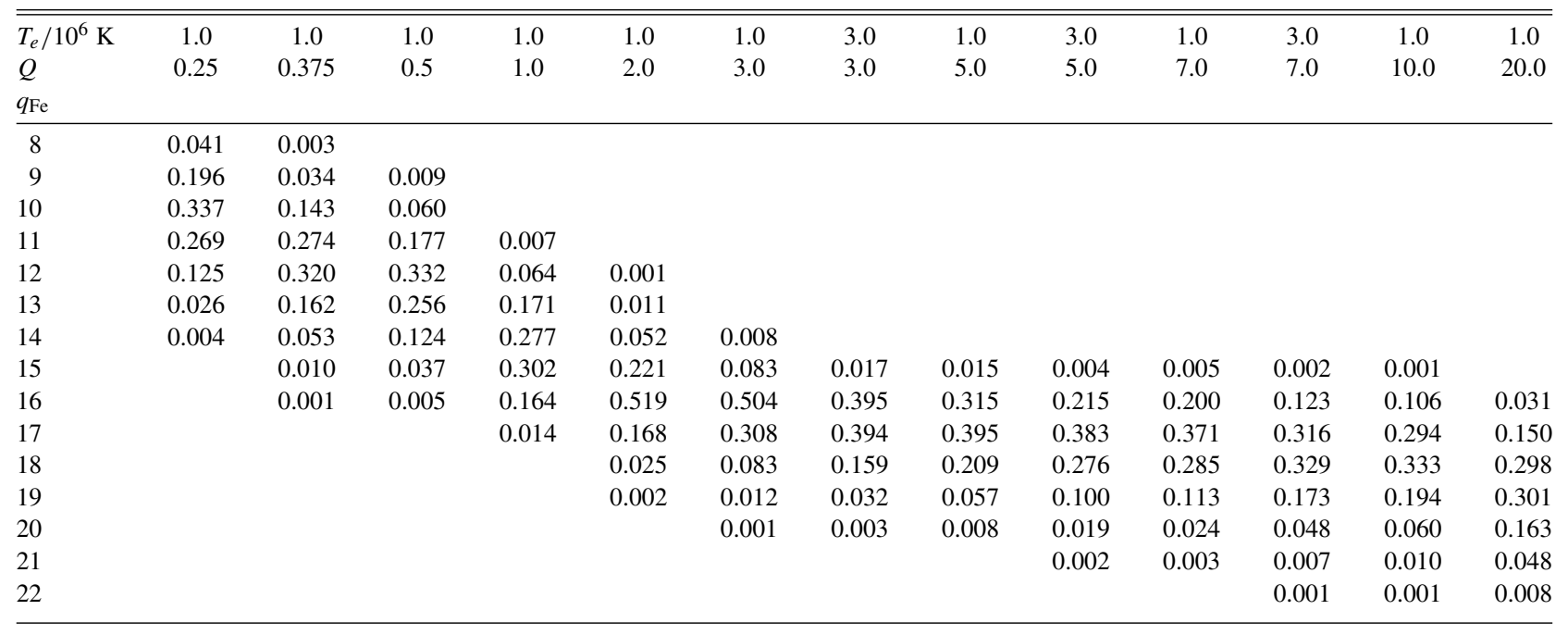

Table 2

Oxygen Charge States with Heating During the Eruption

\begin{tabular}{lcccccccccccccccc}
\hline \hline$T_{e} / 10^{6} \mathrm{~K}$ & 1.0 & 1.0 & 1.0 & 1.0 & 1.0 & 1.0 & 3.0 & 1.0 & 3.0 & 1.0 & 3.0 & 1.0 & 1.0 \\
$Q$ & 0.25 & 0.375 & 0.5 & 1.0 & 2.0 & 3.0 & 3.0 & 5.0 & 5.0 & 7.0 & 7.0 & 10.0 & 20.0 \\
$q_{O}$ & & & & & & & & & \\
\hline 5 & 0.003 & 0.002 & 0.002 & 0.001 & & & & & \\
6 & 0.969 & 0.938 & 0.910 & 0.792 & 0.578 & 0.423 & 0.041 & 0.270 & 0.024 & 0.172 & 0.016 & 0.118 & 0.051 \\
7 & 0.027 & 0.059 & 0.087 & 0.199 & 0.380 & 0.485 & 0.309 & 0.549 & 0.268 & 0.552 & 0.235 & 0.527 & 0.434 \\
8 & & 0.001 & 0.001 & 0.008 & 0.042 & 0.092 & 0.650 & 0.180 & 0.708 & 0.276 & 0.750 & 0.355 & 0.515 \\
\hline
\end{tabular}


Section 1/6 (Ulysses), $<Q_{0}>=6.36$

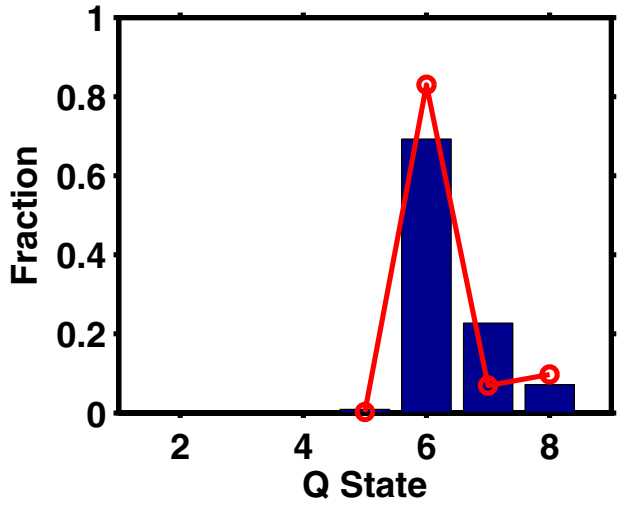

Section $3 / 6$ (Ulysses), $<Q_{0}>=6.60$

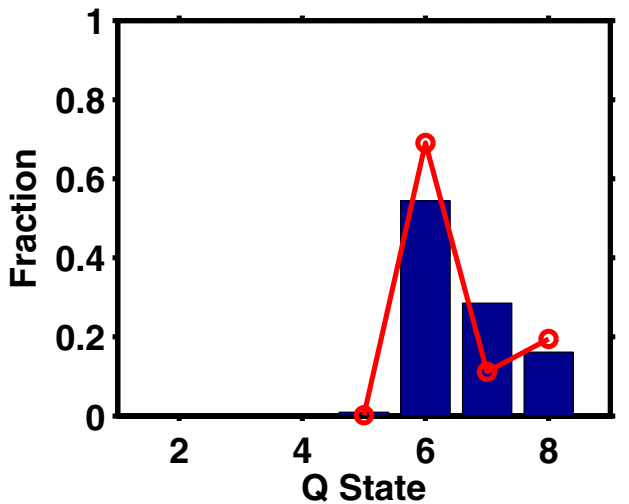

Section 5/6 (Ulysses), $<Q_{0}>=7.15$

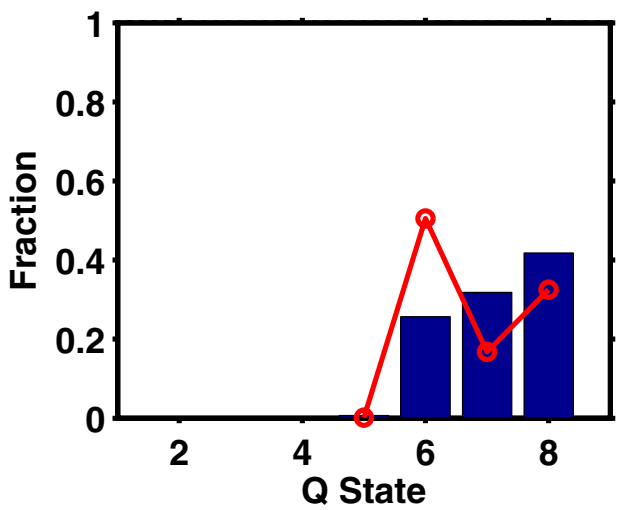

Section 2/6 (Ulysses), $<Q_{0}>=6.91$

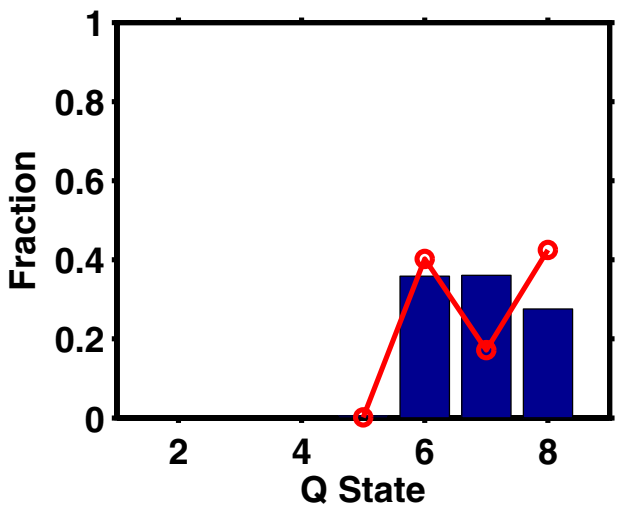

Section 4/6 (Ulysses), $<Q_{0}>=7.52$

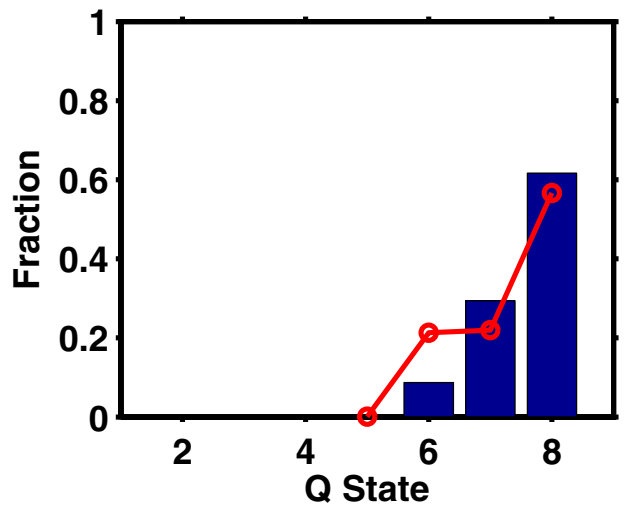

Section 6/6 (Ulysses), $<Q_{0}>=6.73$

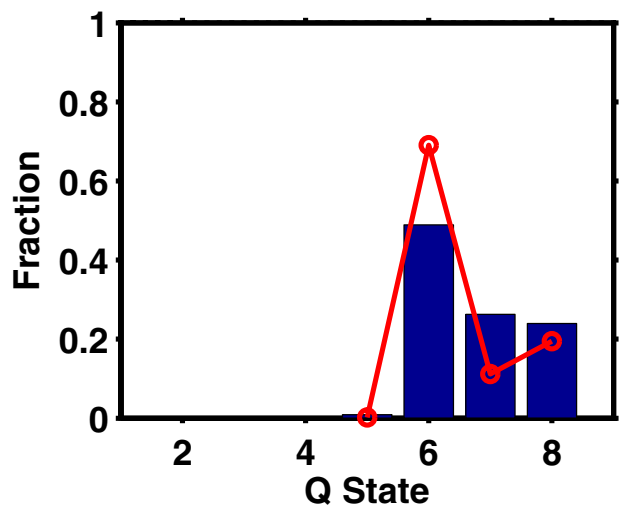

Figure 12. Same as Figure 10, but for six intervals during the Ulysses ICME observation.

(A color version of this figure is available in the online journal.)

Table 3

Carbon Charge States with Heating During the Eruption

\begin{tabular}{lccccccccccccccc}
\hline \hline$T_{e} / 10^{6} \mathrm{~K}$ & 1.0 & 1.0 & 1.0 & 1.0 & 1.0 & 1.0 & 3.0 & 1.0 & 3.0 & 1.0 & 3.0 & 1.0 & 1.0 \\
$Q$ & 0.25 & 0.375 & 0.5 & 1.0 & 2.0 & 3.0 & 3.0 & 5.0 & 5.0 & 7.0 & 7.0 & 10.0 & 20.0 \\
$q_{C}$ & & & & & & & & & & \\
\hline 4 & 0.247 & 0.002 & 0.165 & 0.078 & 0.020 & 0.007 & & 0.002 & \\
5 & 0.587 & 0.599 & 0.600 & 0.555 & 0.403 & 0.286 & 0.008 & 0.176 & 0.005 & 0.112 & 0.003 & 0.079 & 0.038 \\
6 & 0.166 & 0.202 & 0.235 & 0.367 & 0.576 & 0.708 & 0.992 & 0.822 & 0.995 & 0.888 & 0.997 & 0.921 & 0.962 \\
\hline
\end{tabular}

best fit the observations is overlaid. The charge states calculated from the model assume a single initial temperature of $1 \mathrm{MK}$ and additional heating during the eruption, with $Q=0.375$ for Sections $1-3$ and $Q=0.25$ for Section 4. While the exact shape of the distribution is not perfectly replicated, the model does a good job matching the peak charge states. These values for initial temperature and additional heating, $Q$, are used as well for $\mathrm{C}$, $\mathrm{O}$ observed by $A C E$. 

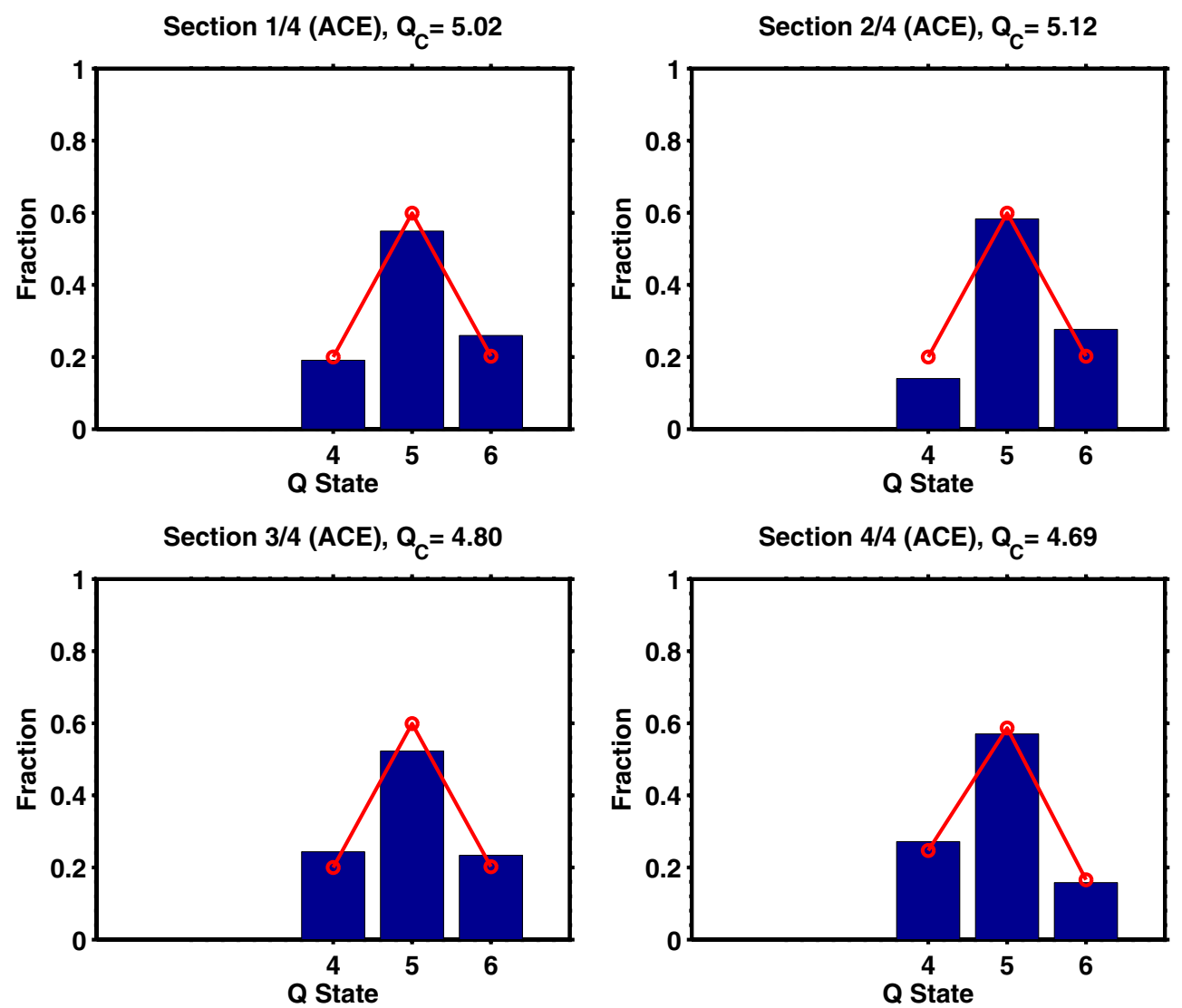

Figure 13. Final $\mathrm{C}$ charge states as measured by $A C E$ for four time intervals during the ICME passage.

(A color version of this figure is available in the online journal.)

Table 4

Ulysses Fe Data

\begin{tabular}{lcccc}
\hline \hline Section & DOY & $Q$ & Warm & Hot \\
\hline 1 & $27.5-29$ & 3 & $85 \%$ & $15 \%$ \\
2 & $29-30$ & 5 & $40 \%$ & $60 \%$ \\
3 & $30-31.5$ & 3 & $70 \%$ & $30 \%$ \\
4 & $31.5-36$ & 5 & $20 \%$ & $80 \%$ \\
5 & $36-38.5$ & 3 & $50 \%$ & $50 \%$ \\
6 & $38.5-40.5$ & 3 & $70 \%$ & $30 \%$ \\
\hline
\end{tabular}

Figure 10 shows the Fe charge state distributions (blue bars) in six sections of the same ICME, observed by Ulysses SWICS. The sections are chosen to highlight particular physical features within the ICME, and are listed in Table 4. Here a bimodal distribution is clear with peaks at $\mathrm{Fe}^{10+}$ or $\mathrm{Fe}^{9+}$ and $\mathrm{Fe}^{17+}$ or $\mathrm{Fe}^{16+}$. All six sections observed at Ulysses show a much higher relative abundance of the high charge states than seen by $A C E$ up to $\mathrm{Fe}^{20+}$. Here, the model used to fit the charge state distributions includes a combination of two plasmas added together. The cooler plasma, which matches the lower charge state peak around $\mathrm{Fe}^{10+}$, is the same plasma that was used to model the $A C E$ observations with the same $Q$ and an initial temperature around $1 \mathrm{MK}$. The hotter plasma starts at $3 \mathrm{MK}$ and was heated further during the eruption with a heating ratio of $Q=3-5$ for the six successive sections, again listed in Table 4 as the "Hot" contribution. These values are used as well for $\mathrm{C}, \mathrm{O}$ observed by Ulysses. These combinations match the observations quite well with the exception of the first section which appears to have a cooler component than the model includes.
Figures 11 through 14 show the oxygen and carbon charge states observed by $A C E$ and Ulysses. The $A C E$ data show dominant charge states of $\mathrm{O}^{6+}$ and $\mathrm{C}^{5+}$, with some $\mathrm{C}^{4+}$ and $\mathrm{C}^{6+}$ throughout the event. The Ulysses data show dominant charge states of $\mathrm{O}^{8+}$ and $\mathrm{C}^{6+}$, with a substantial fraction of $\mathrm{O}^{7+}$ throughout most of the event, more highly ionized than shown in Figure 8, where the heating started from $1 \mathrm{MK}$.

None of the models in Table 1 show a bimodal distribution of Fe charge states. It is possible to find bifurcated Fe charge distributions for a single plasma that is initially hot and then recombines during the eruption. The slowest recombination rates are those for $\mathrm{Fe}^{16+}$ recombining to $\mathrm{Fe}^{15+}$, and for $\mathrm{Fe}^{8+}$ recombining to $\mathrm{Fe}^{7+}$, and so in such circumstances population tends to "bottleneck" (Rakowski et al. 2007) in these two charge states, leading to bifurcated charge state distributions. We can see this in Table 5 where we have shown the $\mathrm{Fe}$ distributions for initial temperatures between 2 and $5 \times 10^{6} \mathrm{~K}$ with either no heating during the eruption or a small amount. With no additional heating we can see peaks at $\mathrm{Fe}^{8+}, \mathrm{Fe}^{12+}$, and $\mathrm{Fe}^{16+}$, depending on the initial temperature. Even a small amount of heating tends to reduce or erase this bimodality. By temperatures of $5 \times 10^{6} \mathrm{~K}$ very little of the low charge states remain, and yet no appreciable $\mathrm{Fe}^{19+}$ or $\mathrm{Fe}^{20+}$ has been produced. Despite the observed Fe charge state distribution at Ulysses exhibiting a bimodal nature, it is not well fit by any of these profiles.

Tables 6 and 7 show the oxygen and carbon charge states for the models starting from a high temperature. The carbon charge states are all too high to match the $A C E$ data, but may be a reasonable match for Ulysses. Temperatures of 3 to $4 \times 10^{6} \mathrm{~K}$ 

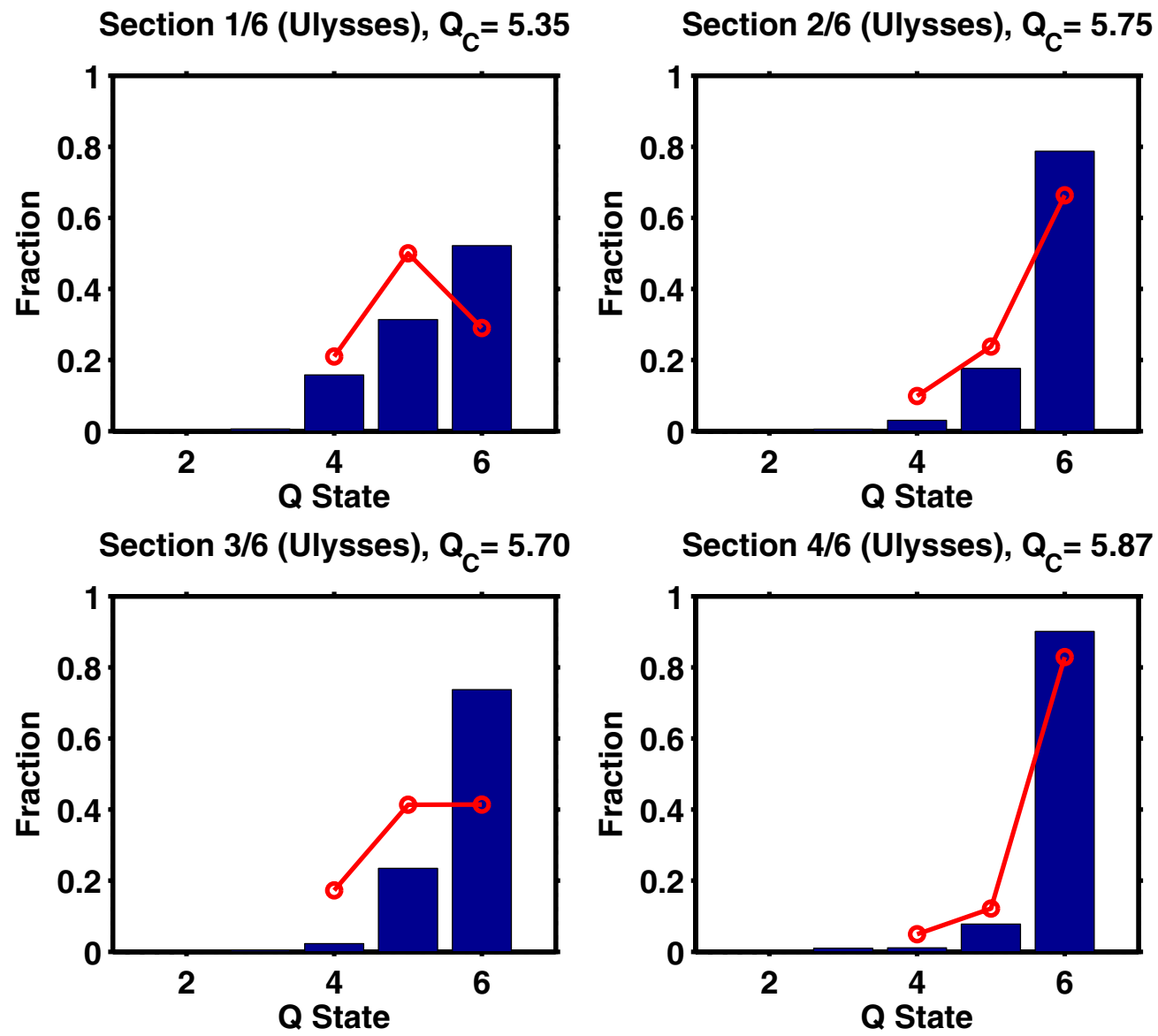

Section $5 / 6$ (Ulysses), $Q_{C}=5.77$
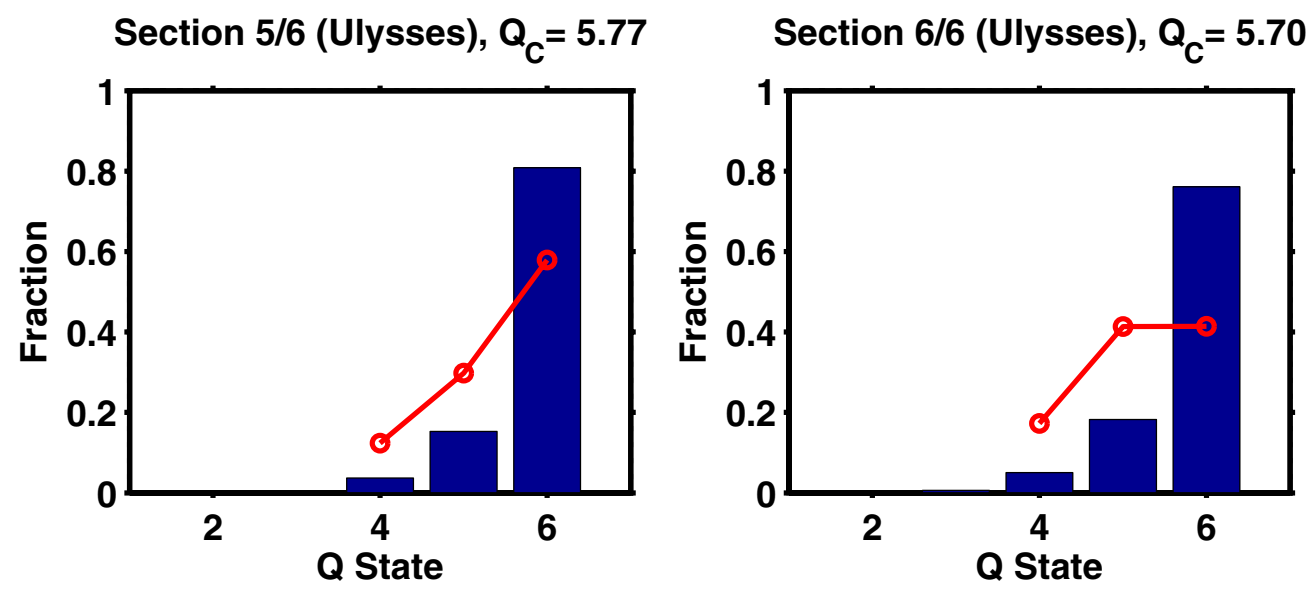

Figure 14. Same as Figure 12, but for six intervals during the Ulysses ICME observation.

(A color version of this figure is available in the online journal.)

are a reasonable match to the oxygen charge states for Ulysses, by $5 \times 10^{6} \mathrm{~K}$ oxygen is overionized.

The $A C E$ data can be reasonably well matched with an initial temperature of $1 \times 10^{6} \mathrm{~K}$ and a heating input of $0.25-0.5$ times the kinetic energy input. The Ulysses data, with that bifurcated $\mathrm{Fe}$ charge distribution, high $\mathrm{Fe}$ charge states up to $\mathrm{Fe}^{20+}$, combined with the persistence of $\mathrm{O}^{7+}$ are harder to match with a single plasma even with a high initial temperature. It is possible to get a bimodal Fe distribution by starting with a hot plasma and letting it recombine, but initial temperatures of $5 \times 10^{6} \mathrm{~K}$ or higher are excluded by the oxygen data and yet are still not hot enough to exhibit $\mathrm{Fe}^{19+}$. Thus we believe that the bimodality in this case must arise due to mixing of a $\sim 1 \mathrm{MK}$ and $\sim 3 \mathrm{MK}$ plasma. Allowing some fraction of the plasma to be heated at a rate of 3-7 times the kinetic energy input and the rest to only have been heated at a rate of $0.25-0.5$ was shown above to reproduce the charge states observed in Ulysses.

Gruesbeck et al. (2011) argue that such mixing is unlikely (if diffusive in nature) and that one can always find a heating function that will reproduce a bimodal distribution, as found for example in postprocessing the MHD simulations of Lynch et al. (2011). Lynch et al. (2011) performed a flux-cancellation model calculation with Magnetohydrodynamics-on-A-Sphere (MAS), which resembles the model of Lin \& Forbes (2000), and a break-out model (Antiochos 1998; Antiochos et al. 1999) calculation with "A Research Code" (ARC7). An important 
Table 5

Iron Charge States from High Initial Temperature

\begin{tabular}{lcccccccc}
\hline \hline$T_{e} / 10^{6} \mathrm{~K}$ & 2.0 & 2.0 & 3.0 & 3.0 & 4.0 & 4.0 & 5.0 & 5.0 \\
$Q$ & 0.0 & 0.25 & 0.0 & 0.25 & 0.0 & 0.25 & 0.0 & 0.25 \\
$Q_{\mathrm{Fe}}$ & & & & & & & & \\
\hline 6 & 0.011 & & & & & & & \\
7 & 0.062 & & 0.003 & & & & & \\
8 & 0.240 & 0.003 & 0.024 & & 0.004 & & 0.001 & \\
9 & 0.079 & 0.023 & 0.019 & & 0.005 & & 0.002 & \\
10 & 0.082 & 0.083 & 0.028 & 0.006 & 0.010 & & 0.004 & \\
11 & 0.169 & 0.181 & 0.080 & 0.025 & 0.035 & 0.006 & 0.017 & 0.002 \\
12 & 0.196 & 0.278 & 0.187 & 0.081 & 0.115 & 0.028 & 0.069 & 0.011 \\
13 & 0.027 & 0.178 & 0.074 & 0.108 & 0.071 & 0.057 & 0.058 & 0.031 \\
14 & 0.028 & 0.099 & 0.098 & 0.151 & 0.111 & 0.122 & 0.103 & 0.089 \\
15 & 0.016 & 0.051 & 0.070 & 0.167 & 0.088 & 0.190 & 0.092 & 0.180 \\
16 & 0.087 & 0.103 & 0.406 & 0.445 & 0.514 & 0.544 & 0.540 & 0.567 \\
17 & & 0.001 & 0.010 & 0.016 & 0.044 & 0.049 & 0.103 & 0.104 \\
18 & & & & & 0.001 & 0.024 & 0.009 & 0.013 \\
19 & & & & & & 0.002 & 0.001 & 0.003 \\
\hline
\end{tabular}

difference between these two simulations was that the MAS model included ohmic heating due to anomalous resistivity at the current sheet whereas the ARC7 model, being ideal MHD, did not. The flux-cancellation CME with ohmic heating produced a significant contribution of high charge states of $\mathrm{Fe}^{16+}$ (compared to Rakowski et al. 2007) while the break-out CME, without anomalous resistivity, failed to produce high charge states within the flux rope. This preliminary work shows that electron heating at the current sheet, such as that provided by an anomalous resistivity, is a necessary component of the energy budget in order to reproduce the high charge states seen in situ.

It is clear for this particular CME that the bimodal charge state distributions observed could not be fit by rapid heating and expansion alone. The density required by Gruesbeck's model is much higher and his speeds are much lower than for this event, hence the freeze-in in his model occurs much further out. The closer-in freeze-in in our work implies that there is not sufficient time for recombination to occur, hence the lower charge states reflect temperatures producing ionization versus recombination. Furthermore, while our nominal density, initial velocity, and acceleration rate were chosen to match the data as well as possible we have experimented with other values for these parameters to see if our results were overly sensitive to these particular choices. Values from $1 / 2$ to 4 times the nominal values were simulated and no bimodality or other qualitative differences were found. The exact values of $Q$ needed to reproduce the higher charge states were different by factors of at most two. We have taken great care here to consider the CME evolution for this particular event by connecting the plasma heating to the CME acceleration. The heating illustrated in Figures 6 and 7 occurs while the CME ejecta are within about $2 R_{\odot}$, which would place the CME shock within about $3 R_{\odot}$. Figures 9 and 10 in Grechnev et al. (2008) show that the peak GOES X-ray temperature and energy input to the flare correspond temporally to this shock position.

So, while bimodal Fe distributions can be obtained for a single plasma with specific heating profiles, a bimodal charge state distribution is also good evidence for mixing of two or more distinct plasmas during the CME eruption, especially when the density is lower and the expansion is faster. This mixing is supported by the observations at Ulysses which include periods with only the cooler plasma, only the hotter plasma, and the simultaneous presence of both plasmas. During time intervals
Table 6

Oxygen Charge States from High Initial Temperature

\begin{tabular}{lcccccccc}
\hline \hline$T_{e} / 10^{6} \mathrm{~K}$ & 2.0 & 2.0 & 3.0 & 3.0 & 4.0 & 4.0 & 5.0 & 5.0 \\
$Q$ & 0.0 & 0.25 & 0.0 & 0.25 & 0.0 & 0.25 & 0.0 & 0.25 \\
$Q_{O}$ & & & & & & & & \\
\hline 5 & 0.013 & 0.001 & 0.002 & & & & & \\
6 & 0.525 & 0.514 & 0.118 & 0.110 & 0.029 & 0.026 & 0.011 & 0.009 \\
7 & 0.396 & 0.413 & 0.380 & 0.367 & 0.208 & 0.186 & 0.131 & 0.108 \\
8 & 0.065 & 0.071 & 0.500 & 0.523 & 0.762 & 0.788 & 0.858 & 0.882 \\
\hline
\end{tabular}

Table 7

Carbon Charge States from High Initial Temperature

\begin{tabular}{lcccccccc}
\hline \hline$T_{e} / 10^{6} \mathrm{~K}$ & 2.0 & 2.0 & 3.0 & 3.0 & 4.0 & 4.0 & 5.0 & 5.0 \\
$Q$ & 0.0 & 0.25 & 0.0 & 0.25 & 0.0 & 0.25 & 0.0 & 0.25 \\
$Q_{C}$ & & & & & & & & \\
\hline 4 & 0.010 & 0.008 & 0.002 & 0.001 & & & & \\
5 & 0.135 & 0.107 & 0.060 & 0.039 & 0.040 & 0.023 & 0.031 & 0.017 \\
6 & 0.855 & 0.885 & 0.937 & 0.960 & 0.959 & 0.976 & 0.968 & 0.983 \\
\hline
\end{tabular}

when both plasmas appear simultaneously, this is true down to the $6 \mathrm{hr}$ time resolution of the Ulysses observations and is particularly evident in Figure 2 from DOY 36-39.

We suggest that such mixing arises from instabilities and processes associated with reconnection. Drake et al. (2006) argue that electron acceleration in reconnection occurs in contracting magnetic islands in the reconnection outflow. Such a geometry would naturally lead to strongly and less strongly heated regions within the outflow. Oka et al. (2010) offer a slightly different view, where magnetic islands formed in the outflow coalesce, accelerating electrons at these secondary reconnection sites. Again, the electron heating or acceleration is not uniform over the volume of the current sheet. The split between hotter and cooler plasma in modeling the Fe charge states observed by Ulysses is then readily interpretable in terms of the relative volume of reconnecting plasma occupied by magnetic islands. Section 4 in the Ulysses data, dominated by the strongly heated Fe plasma, might be analogous to the observations of Krucker et al. (2010), where an accelerated electron fraction of close to $100 \%$ was observed in an "above the loop top" source during the 2007 December 31 flare. Another possibility might be the mixing of hot and warm plasma as the flux rope is formed by reconnection before or during the eruption, as in the break-out or flux-cancellation models (Lynch et al. 2011), though the $6 \mathrm{hr}$ cadence would limit the "coarseness" of such a process.

\section{CONCLUSIONS}

The 2005 January 20 event was observed by both $A C E$ and Ulysses SWICS instruments. ACE, which observed the flank of the ICME, sees charge states of $\mathrm{O}^{6+}$ and $\mathrm{C}^{5+}$ indicative of an initial temperature of $\sim 1 \times 10^{6} \mathrm{~K}$. The Fe charge state distribution at $A C E$ can be explained with heating during the eruption at a rate of $0.25-0.5$ times the kinetic energy input, with charge states freezing in further out than is the case for $\mathrm{O}$ or C. Ulysses passed through a more central region of the ICME and observed higher charge states of $\mathrm{O}^{8+}, \mathrm{C}^{6+}$ and as high as $\mathrm{Fe}^{20+}$. The observations of hot material in the central regions of the CME have been supported by the work of Reinard (2008) and the flux-cancellation MHD and ionization model in Lynch et al (2011). The Fe charge state distribution is both broad and bimodal. Starting the plasma from an initial high temperature 
can produce bimodality as the ions recombine. However, we were not able to reproduce both the presence of $\mathrm{O}^{7+}$ and a range of low Fe charge states while also obtaining the highest Fe charge states with one plasma history as the initial densities and speed of the ICME were too fast to allow recombination to occur before freeze-in. We therefore postulate that in Ulysses we are seeing a combination of plasma that was heated similarly to that in $A C E$ (0.25-0.5 times the kinetic energy input) with hotter plasma that was heated as much as five times the kinetic energy input. The fact that a combination of plasma very similar to that observed at $A C E$ and a plasma heated to a high initial temperature of $3 \mathrm{MK}$ matches the observation at Ulysses provides substantial support for the idea of mixing of multiple plasmas at different temperatures during the eruption. We suggest that this apparent mixing of plasmas of different temperatures could be interpreted in terms of the plasmoid instability in the reconnection outflow. Electrons are strongly heated in the magnetic islands, leading to regions of hot and highly ionized plasma embedded in less heated surroundings.

This work has been supported by NASA LWS Grant NNH05AA05I (J.M.L. and C.E.R.) and by an NSF SHINE Postdoctoral Fellowship ATM-0523998 (S.T.L.).

\section{REFERENCES}

Antiochos, S. K. 1998, ApJ, 502, L181

Antiochos, S. K., DeVore, C. R., \& Klimchuk, K. A. 1999, ApJ, 510, 485

Bame, S. J., Asbridge, J. R., Feldman, W. C., \& Kearney, P. D. 1974, Sol. Phys., 35,137

Berkebile-Stoiser, S., Veronig, A. M., Bein, B. M., \& Temmer, M. 2012, ApJ, 753, 88

Borrini, G., Gosling, J. T., Bame, S. J., \& Feldman, W. C. 1982, J. Geophys. Res., 87, 7370

Bryans, P., Badnell, N. R., Gorczyca, T. W., et al. 2006, ApJS, 167, 343

Bürgi, A., \& Geiss, J. 1986, Sol. Phys., 103, 347

Burlaga, L. F. 1991, in Physics of the Inner Heliosphere, Vol. 2, ed. R. Schwenn \& E. Marsch (Berlin: Springer), 1

Burlaga, L. F., Skoug, R. M., Smith, C. W., et al. 2001, J. Geophys. Res., 106, 20957

Cane, H. V., Mewaldt, R. A., Cohen, C. M. S., \& von Rosenvinge, T. T. 2006, J. Geophys. Res., 111, A06S90

Cane, H. V., \& Richardson, I. G. 2003, J. Geophys. Res. A, 108, 1156

Chen, Y., Esser, R., \& Hu, Y. 2003, ApJ, 582, 467

Drake, J. F., Swisdak, M., Che, H., \& Shay, M. A. 2006, Nature, 443, 553

Edmiston, J. P., \& Kennel, C. F. 1984, J. Plasma. Phys., 32, 429

Esser, R., \& Edgar, R. J. 2001, ApJ, 563, 1055

Esser, R., \& Edgar, R. J. 2002, Adv. Space Res., 30, 481

Farris, M. H., \& Russell, C. T. 1994, J. Geophys. Res., 99, 17681

Farrugia, C. J., Popecki, M., Möbius, E., et al. 2002, J. Geophys. Res. A, 107,1240

Fenimore, E. E. 1980, ApJ, 235, 245

Foullon, C., Owen, C. J., Dasso, S., et al. 2007, Sol. Phys., 244, 139

Galvin, A. B., \& Gloeckler, G. 1997, in Proc. 31st ESLAB Symp., Correlated Phenomena at the Sun, in the Heliosphere and in Geospace, ed. A. Wilson (ESA SP-415; Noordwijk: ESA), 323

Geiss, J., Gloeckler, G., von Steiger, R., et al. 1995, Science, 268, 1033

Gloeckler, G., Cain, J., Ipavich, F. M., et al. 1998, Space Sci. Rev., 86,497

Gloeckler, G., Fisk, L. A., Hefti, S., et al. 1999, Geophys. Res. Lett., 26,157

Gloeckler, G., Geiss, J., Balsiger, H., et al. 1992, A\&AS, 92, 267

Gopalswamy, N., Xie, H., Yashiro, S., \& Usoskin, I. 2005, in Proc. 29th Int. Cosmic Ray Conf., ed. B. Sripathi Acharya, S. Gupta, P. Jagadeesan,
A. Jain, S. Karthikeyan, S. Morris, \& S. Tonwar (Mumbai: Tata Institute of Fundamental Research), 169

Gopalswamy, N., \& Yashiro, S. 2011, ApJ, 736, L17

Gosling, J. T. 1990, in Physics of Magnetic Flux Ropes, ed. E. R. Priest \& L. C. Lee (Geophys. Monogr. Ser. 58; Washington, DC: AGU), 343

Gosling, J. T., \& Riley, P. 1996, Geophys. Res. Lett., 23, 2867

Grechnev, V. V., Kurt, V. G., Chertok, I. M., et al. 2008, Sol. Phys., 252, 149

Gruesbeck, J. R., Lepri, S. T., Zurbuchen, T. H., \& Antiochos, S. K. 2011, ApJ, 730,103

Haggerty, D. K., Roelof, E. C., Smith, C. W., et al. 2000, in AIP Conf. Proc. 528, Acceleration and Transport of Energetic Particles Observed in the Heliosphere: ACE 2000, ed. R. A. Mewaldt, M. Miller, J. R. Jokipii et al. (Melville, NY: AIP), 266

Henke, T., Woch, J., Mall, U., et al. 1998, Geophys. Res. Lett., 25, 3465

Henke, T., Woch, J., Schwenn, R., et al. 2001, J. Geophys. Res., 106, 10597

Hundhausen, A. J. 1968, Space Sci. Rev., 8, 690

Hundhausen, A. J. 1970, Rev. Geophys., 8, 729

Ko, Y.-K., Raymond, J. C., Li, J., et al. 2002, ApJ, 578, 979

Krucker, S., Hudson, H. S., Glesener, L., et al. 2010, ApJ, 714, 1108

Laming, J. M. 2004, ApJ, 604, 874

Laming, J. M., \& Grun, J. 2002, Phys. Rev. Lett., 89, 125002

Laming, J. M., \& Grun, J. 2003, Phys. Plasmas, 10, 1614

Laming, J. M., \& Hwang, U. 2003, ApJ, 597, 347

Laming, J. M., \& Lepri, S. T. 2007, ApJ, 660, 1642

Lepri, S. T., \& Zurbuchen, T. H. 2004a, J. Geophys. Res. A, 109, 1112

Lepri, S. T., \& Zurbuchen, T. H. 2004b, J. Geophys. Res. A, 109, 6101

Lepri, S. T., Zurbuchen, T. H., Fisk, L. A., et al. 2001, J. Geophys. Res., 106,29231

Lin, J., \& Forbes, T. G. 2000, J. Geophys. Res., 105, 2375

Lynch, B. J., Reinard, A. A., Mulligan, T., et al. 2011, ApJ, 740, 112

Lynch, B. J., Zurbuchen, T. H., Fisk, L. A., \& Antiochos, S. K. 2003, J. Geophys. Res. A, 108, 1239

Mewaldt, R. A., Looper, M. D., Cohen, C. M. S., et al. 2005, in Proc. 29th Int. Cosmic Ray Conf., ed. B. Sripathi Acharya, S. Gupta, P. Jagadeesan, A. Jain, S. Karthikeyan, S. Morris, \& S. Tonwar (Mumbai: Tata Institute of Fundamental Research), 101

Neugebauer, M., Goldstein, R., \& Goldstein, B. E. 1997, J. Geophys. Res., 102,19743

Oka, M., Phan, T.-D., Krucker, S., Fujimoto, M., \& Shinohara, I. 2010, ApJ, 714,915

Owocki, S. P., Holzer, T. E., \& Hundhausen, A. J. 1983, ApJ, 275, 354

Rakowski, C. E., Laming, J. M., \& Lepri, S. T. 2007, ApJ, 667, 602

Rakowski, C. E., Laming, J. M., \& Lyutikov, M. 2011, ApJ, 730, 30

Reinard, A. A. 2005, ApJ, 620, 501

Reinard, A. A. 2008, ApJ, 682, 1289

Reinard, A. A., Zurbuchen, T. H., Fisk, L. A., et al. 2001, in AIP Conf. Proc. 598, Solar and Galactic Composition, ed. R. Wimmer-Schweingruber (Melville, NY: AIP), 139

Rodriguez, L., Zhukov, A. N., Dasso, S., et al. 2008, Ann. Geophys., 26, 213

Rouillard, A. P., Odstrčil, D., Sheeley, N. R., Jr., et al. 2011, ApJ, 735, 7

Russell, C. T., \& Mulligan, T. 2002, Plan. Space. Sci., 50, 527

Savani, N. P., Shiota, D., Kusano, K., Lugaz, N., \& Vourlidas, A. 2012, ApJ, 759,103

Schrijver, C. J. 2001, ApJ, 547, 475

Schrijver, C. J., \& DeRosa, M. L. 2003, Sol. Phys., 212, 165

Simnett, G. 2006, A\&A, 445, 715

Skoug, R. M., Feldman, W. C., Gosling, J. T., McComas, D. J., \& Smith, C. W. 2000, J. Geophys. Res., 105, 23069

Stone, E. C., Frandsen, A. M., Mewaldt, R. A., et al. 1998, Space Sci. Rev., 86,1

Wenzel, K.-P., Marsden, R. G., Page, D. E., \& Smith, E. J. 1992, A\&AS, 92, 207

Wilhelm, K., Inhester, B., \& Newmark, J. S. 2002, A\&A, 382, 328

Zurbuchen, T. H., Fisk, L. A., Lepri, S. T., \& von Steiger, R. 2003, in AIP Conf. Proc. 679, Tenth International Solar Wind Conference, ed. M. Velli, R. Bruno, \& F. Malara (Melville, NY: AIP), 604

Zurbuchen, T. H., Gloeckler, G., Ipavich, F., et al. 2004, Geophys. Res. Lett., 31,11805

Zurbuchen, T. H., \& Richardson, I. G. 2006, Space Sci. Rev., 123, 31 